
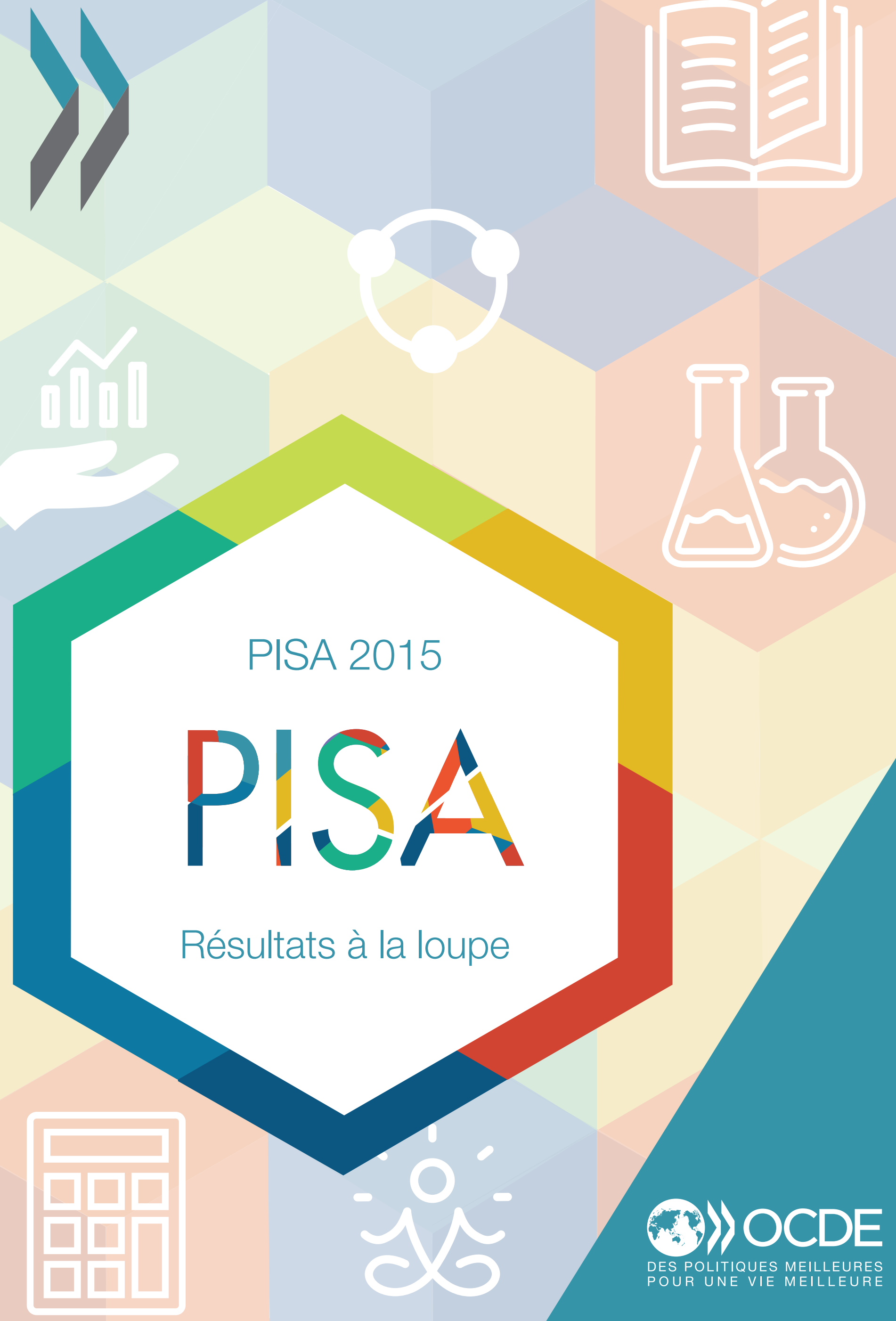


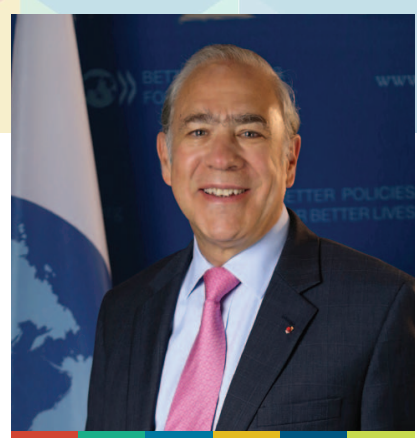

En dix ans, le Programme international de l'OCDE pour le suivi des acquis des élèves (PISA) est devenu la référence mondiale dans le domaine de l'évaluation de la qualité, de l'équité et de l'efficience des systèmes d'éducation. En identifiant les caractéristiques des systèmes d'éducation très performants, l'enquête PISA permet aux gouvernements et aux professionnels de l'éducation de s'inspirer de politiques efficaces qu'ils peuvent ensuite adapter à leur contexte local.

La dernière évaluation PISA en date, celle de 2015, s'est concentrée sur la science. De la prise d'un antidouleur à la préparation d'un repas " équilibré ", en passant par la consommation de lait pasteurisé ou l'achat d'une voiture hybride, la science est omniprésente dans nos vies. Les tubes à essai, le tableau périodique sont autant d'images d'Épinal de la science. Pourtant, c'est à la science que nous devons la quasi-totalité des instruments qui existent dans le monde, du simple ouvre-boîte à la sonde spatiale la plus sophistiquée. Et, point important s'il en est, la science n'est pas la chasse gardée des scientifiques. À l'heure de l'afflux massif d'informations, à un moment où le monde évolue rapidement, nous devons tous être capables de "réfléchir comme des scientifiques " : de jauger les faits pour parvenir à une conclusion ; et de comprendre que la "vérité » scientifique d'aujourd'hui ne sera peut-être pas celle de demain grâce aux nouvelles découvertes et à l'amélioration de la compréhension des forces naturelles et du potentiel et des limites de la technologie.

Cette brochure présente certains des résultats de l'enquête PISA 2015. II en ressort que tous les pays peuvent s'améliorer, même les plus performants. Dans un monde où les taux de chômage sont élevés chez les jeunes, où les inégalités se creusent, où de fortes disparités persistent entre les sexes et où la croissance doit se faire inclusive dans de nombreux pays, il apparaît urgent de prendre des mesures concrètes pour offrir à tous les élèves la meilleure éducation possible.
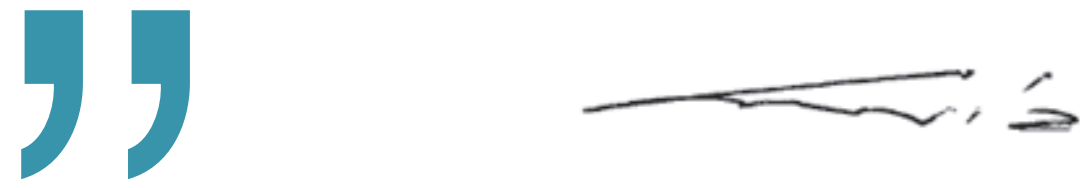

Angel Gurría

Secrétaire général de l'OCDE 


\section{Qu'est-ce que l'enquête PISA ?}

"Qu'importe-t-il de savoir et de savoir faire en tant que citoyen ? " C'est pour répondre à cette question et au besoin de données comparables à l'échelle internationale sur la performance des élèves que l'Organisation de coopération et de développement économiques (OCDE) a mis sur pied l'évaluation des élèves âgés de 15 ans qui a lieu tous les trois ans dans le monde, et que l'on connait sous le nom de Programme international pour le suivi des acquis des élèves, ou PISA. L'enquête PISA évalue dans quelle mesure les élèves qui approchent du terme de leur scolarité obligatoire possèdent certaines des connaissances et compétences essentielles pour participer pleinement à la vie de nos sociétés modernes. L'enquête se concentre sur des matières clés des programmes scolaires, à savoir les sciences, la compréhension de l'écrit et les mathématiques. Les compétences des élèves sont également évaluées dans un domaine novateur (la résolution collaborative de problèmes, en 2015). L'enquête PISA ne cherche pas simplement à évaluer la faculté des élèves à reproduire ce qu'ils ont appris, mais vise aussi à déterminer dans quelle mesure ils sont capables de se livrer à des extrapolations à partir de ce qu'ils ont appris et d'utiliser leurs connaissances dans des situations qui ne leur sont pas familières, qu'elles soient ou non en rapport avec l'école. Cette approche reflète le fait que les économies modernes valorisent davantage la capacité des individus à utiliser leurs connaissances, plutôt que ces connaissances en tant que telles.

L'enquête PISA est un programme de longue haleine qui permet d'éclairer les politiques et les pratiques dans le domaine de l'éducation ; elle aide à suivre l'évolution de l'acquisition de connaissances et de compétences par les élèves dans les pays participants, ainsi que dans différents sous-groupes de la population au sein même des pays. Les résultats de l'enquête PISA identifient les compétences des élèves dans les pays les plus performants et dans les systèmes d'éducation qui progressent le plus rapidement pour révéler tout le potentiel de l'éducation. Les décideurs du monde entier peuvent utiliser ces résultats pour comparer les connaissances et compétences de leurs élèves à celles des élèves des autres pays participants, fixer des objectifs chiffrés d'amélioration en fonction des accomplissements mesurables d'autres pays, et s'inspirer des politiques et pratiques mises en œuvre ailleurs. Si l'enquête PISA ne permet pas d'identifier des relations de cause à effet entre les politiques et pratiques et les résultats des élèves, elle montre aux professionnels de l'éducation, aux décideurs et au grand public en quoi les systèmes d'éducation se ressemblent et se différencient et les implications qui en découlent pour les élèves.

\section{Caractéristiques de l'enquête PISA 2015}

\section{Le contenu}

- Les sciences sont le domaine majeur d'évaluation de l'enquête PISA 2015, dont les domaines mineurs sont la compréhension de l'écrit, les mathématiques et la résolution collaborative de problèmes. Lors de l'enquête PISA 2015, il a également été proposé aux pays et économies d'évaluer la culture financière des élèves, à titre d'option.

\section{Les élèves}

- Au total, environ 540000 élèves, représentatifs des quelque 29 millions d'élèves âgés de 15 ans scolarisés dans les 72 pays et économies participants, ont passé les épreuves PISA en 2015.

\section{Les épreuves}

- Chaque élève a répondu à des épreuves informatisées d'une durée totale de deux heures.

- Les épreuves PISA comportent des questions à choix multiple ainsi que des items demandant aux élèves de formuler leurs propres réponses. Les questions sont regroupées dans des unités qui décrivent une situation s'inspirant de la vie réelle. Au total, des items de sciences, de compréhension de l'écrit, de mathématiques et de résolution collaborative de problèmes, représentant 810 minutes de test environ, ont été administrés, les élèves répondant à des épreuves constituées de différentes combinaisons de ces items.

- Les élèves ont par ailleurs passé 35 minutes à répondre à un questionnaire sur eux-mêmes, leur milieu familial,

leur établissement d'enseignement et leurs expériences concernant leur établissement et leur apprentissage. Les chefs d'établissement ont quant à eux rempli un questionnaire à propos de leur système scolaire et de l'environnement d'apprentissage dans leur établissement. Afin d'élargir la base d'informations, certains pays et économies ont décidé de demander aux enseignants de remplir un questionnaire à leur intention. C'est la première fois qu'il a été proposé aux pays et économies participant à l'enquête PISA d'administrer ce questionnaire aux enseignants à titre d'option. Dans certains pays et économies, un questionnaire, également proposé à titre d'option, a été distribué aux parents d'élèves pour recueillir des informations sur la façon dont ils perçoivent l'établissement de leur enfant et s'y engagent, dont ils soutiennent leur enfant dans son apprentissage à la maison et dont ils perçoivent ses aspirations professionnelles, en particulier en sciences. Les pays et économies avaient également la possibilité d'administrer deux autres questionnaires facultatifs aux élèves : I'un sur la mesure dans laquelle ils sont familiarisés avec les technologies de l'information et de la communication (TIC), et les utilisent ; et le second, sur leur parcours scolaire jusqu'au moment de l'évaluation, y compris les interruptions de ce dernier, ainsi que sur la question de savoir s'ils se préparent à l'exercice d'une profession et, dans l'affirmative, de quelle façon. 


\section{L'excellence et l'équité dans l'éducation}

\section{Que nous apprennent les résultats ?}

\section{La performance des élèves en sciences et leurs attitudes à l'égard de la science}

- En sciences, Singapour devance tous les autres pays et économies participants. Le Japon, l'Estonie, la Finlande et le Canada, listés par ordre décroissant de leur performance moyenne en sciences, sont les quatre pays les plus performants de la zone OCDE.

- En moyenne, environ 8 \% des élèves sont très performants en sciences dans les pays de l'OCDE (et $24 \%$ à Singapour) : ils se classent au niveau 5 ou 6 de compétence. À ces niveaux, les élèves possèdent suffisamment de connaissances et de compétences scientifiques pour les appliquer de manière créative et autonome dans un large éventail de situations, y compris des situations qui ne leur sont pas familières.

- Environ 20 \% des élèves des pays de l'OCDE se situent sous le niveau 2. Au niveau 2, qui est considéré comme le seuil de compétence en culture scientifique, les élèves sont capables de s'appuyer sur des connaissances du contenu et des connaissances procédurales élémentaires pour identifier des explications appropriées, interpréter des données et déterminer la question au cœur d'une expérience scientifique simple. Tous les élèves devraient avoir atteint le niveau 2 à la fin de leur scolarité obligatoire.

- Dans la majorité des pays dont les données sont comparables, la performance en sciences aux épreuves PISA est restée essentiellement identique depuis 2006. Néanmoins, la performance moyenne en sciences a augmenté entre 2006 et 2015 en Colombie, en Israël, à Macao (Chine), au Portugal, au Qatar et en Roumanie. Durant cette période, le pourcentage d'élèves atteignant le niveau 5 ou 6 a augmenté, tandis que le pourcentage d'élèves sous le seuil de compétence (niveau 2) a diminué à Macao (Chine), au Portugal et au Qatar.

- Bien que l'écart de performance en sciences entre les sexes tende à être faible, en moyenne, dans 33 pays et économies, le pourcentage d'élèves très performants en sciences est plus élevé chez les garçons que chez les filles. La Finlande est le seul pays où les filles sont plus susceptibles d'être très performantes que les garçons.

- En moyenne, dans les pays de l'OCDE, 25 \% des garçons et $24 \%$ des filles ont indiqué qu'ils envisageaient d'exercer une profession scientifique. Toutefois, les disciplines scientifiques que les élèves privilégient varient entre les sexes : les filles aspirent plus à exercer une profession en rapport avec la santé, tandis que, dans la quasi-totalité des pays, les garçons s'imaginent plutôt informaticiens, scientifiques ou ingénieurs.

\section{La performance des élèves en compréhension de l'écrit et en mathématiques}

- En moyenne, dans les pays de l'OCDE, 20 \% environ des élèves n'atteignent pas le seuil de compétence en compréhension de l'écrit. Ce pourcentage n'a pas évolué depuis 2009.

- En moyenne, dans les pays de l'OCDE, l'écart de score en compréhension de l'écrit, favorable aux filles, a diminué de 12 points entre 2009 et 2015 : le score a augmenté chez les garçons, en particulier chez les plus performants, mais a diminué chez les filles, en particulier chez les moins performantes.

- À Pékin-Shanghai-Jiangsu-Guangdong (Chine), à Hong-Kong (Chine), à Singapour et au Taipei chinois, plus d'un élève sur quatre est très performant en mathématiques, et se distingue donc par sa capacité à formuler des situations complexes en termes mathématiques, à l'aide de représentations symboliques.

\section{L'équité dans l'éducation}

- Le Canada, le Danemark, l'Estonie, Hong-Kong (Chine) et Macao (Chine) se distinguent par des niveaux élevés de performance, ainsi qu'un rendement de l'éducation plus équitable.

- En moyenne, dans les pays de l'OCDE, les élèves défavorisés sur le plan socio-économique sont près de trois fois plus susceptibles que leurs pairs favorisés de ne pas atteindre le seuil de compétence en sciences. Toutefois, environ $29 \%$ des élèves défavorisés sont considérés comme résilients : ils déjouent les pronostics et obtiennent des scores élevés en sciences. En outre, à Macao (Chine) et au Viet Nam, les élèves les plus défavorisés selon l'échelle internationale devancent les élèves les plus favorisés d'environ 20 autres pays et économies participant à l'enquête PISA.

- Bien qu'entre 2006 et 2015, aucun pays ou économie n'ait amélioré simultanément son niveau de performance en sciences et son degré d'équité, dans neuf pays où le score moyen en sciences est resté stable, le niveau socio-économique est devenu une variable prédictive moins probante de la performance des élèves. C'est aux États-Unis que le degré d'équité a connu la progression la plus remarquable durant cette période.

- En moyenne, dans les pays de l'OCDE, et après contrôle du niveau socio-économique, les élèves issus de l'immigration sont plus de deux fois plus susceptibles que leurs pairs autochtones d'obtenir des résultats inférieurs au seuil de compétence en sciences. Toutefois, $24 \%$ des élèves issus de l'immigration et d'un milieu socio-économique défavorisé sont considérés comme « résilients ».

- En moyenne, dans les pays comptant des effectifs relativement importants d'élèves issus de l'immigration, la scolarisation dans un établissement présentant une forte concentration d'élèves issus de l'immigration n'est pas corrélée à l'obtention de moins bons résultats, après contrôle du profil socio-économique de l'effectif d'élèves dudit établissement. 


\begin{tabular}{|c|c|c|c|c|c|c|c|c|}
\hline & \multicolumn{8}{|c|}{$\begin{array}{l}\text { Pays/économies dont le score moyen/le pourcentage d'élèves très performants sont supérieurs à la moyenne de l'OCDE } \\
\text { Pays/économies dont le pourcentage d'élèves peu performants est inférieur à la moyenne de l'OCDE }\end{array}$} \\
\hline & \multicolumn{8}{|c|}{$\begin{array}{c}\text { Pays/économies dont le score moyen/le pourcentage d'élèves très performants/le pourcentage d'élèves peu performants ne s'écartent pas de la moyenne de l'OCDE } \\
\text { dans une mesure statistiquement significative }\end{array}$} \\
\hline & \multicolumn{8}{|c|}{$\begin{array}{l}\text { Pays/économies dont le score moyen/le pourcentage d'élèves très performants sont inférieurs à la moyenne de l'OCDE } \\
\text { Pays/économies dont le pourcentage d'élèves peu performants est supérieur à la moyenne de l'OCDE }\end{array}$} \\
\hline & \multicolumn{2}{|c|}{ Sciences } & \multicolumn{2}{|c|}{ Compréhension de l'écrit } & \multicolumn{2}{|c|}{ Mathématiques } & Sciences, compréhensio & e l'écrit et mathématiques \\
\hline & 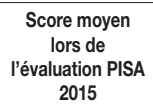 & $\begin{array}{l}\text { Évolution } \\
\text { moyenne sur } \\
3 \text { ans }\end{array}$ & $\begin{array}{c}\text { Score moyen } \\
\text { lors de } \\
\text { l'évaluation PISA } \\
2015\end{array}$ & $\begin{array}{l}\text { Évolution } \\
\text { moyenne sur } \\
3 \text { ans }\end{array}$ & 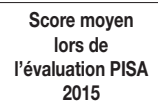 & $\begin{array}{l}\text { Évolution } \\
\text { moyenne sur } \\
3 \text { ans }\end{array}$ & $\begin{array}{l}\text { Pourcentage d'élèves très } \\
\text { performants dans au moins un } \\
\text { domaine d'évaluation (niveau } \\
5 \text { ou } 6 \text { ) }\end{array}$ & $\begin{array}{c}\text { Pourcentage d'élèves peu } \\
\text { performants dans l'ensemble } \\
\text { des trois domaines d'évaluation } \\
\text { (sous le niveau 2) }\end{array}$ \\
\hline & Score moyen & Diff. de score & Score moyen & Diff. de score & Score moyen & Diff. de score & $\%$ & $\%$ \\
\hline Moyenne OCDE & 493 & -1 & 493 & -1 & 490 & -1 & 15.3 & 13.0 \\
\hline Singapour & 556 & 7 & 535 & 5 & 564 & 1 & 39.1 & 4.8 \\
\hline Japon & 538 & 3 & 516 & -2 & 532 & 1 & 25.8 & 5.6 \\
\hline Estonie & 534 & 2 & 519 & 9 & 520 & 2 & 20.4 & 4.7 \\
\hline Taipei chinois & 532 & 0 & 497 & 1 & 542 & 0 & 29.9 & 8.3 \\
\hline Finlande & 531 & -11 & 526 & -5 & 511 & -10 & 21.4 & 6.3 \\
\hline Macao (Chine) & 529 & 6 & 509 & 11 & 544 & 5 & 23.9 & 3.5 \\
\hline Canada & 528 & -2 & 527 & 1 & 516 & -4 & 22.7 & 5.9 \\
\hline Viet Nam & 525 & -4 & 487 & -21 & 495 & -17 & 12.0 & 4.5 \\
\hline Hong-Kong (Chine) & 523 & -5 & 527 & -3 & 548 & 1 & 29.3 & 4.5 \\
\hline P-S-J-G (Chine) & 518 & $\mathrm{~m}$ & 494 & $\mathrm{~m}$ & 531 & $\mathrm{~m}$ & 27.7 & 10.9 \\
\hline Corée & 516 & -2 & 517 & -11 & 524 & -3 & 25.6 & 7.7 \\
\hline Nouvelle-Zélande & 513 & -7 & 509 & -6 & 495 & -8 & 20.5 & 10.6 \\
\hline Slovénie & 513 & -2 & 505 & 11 & 510 & 2 & 18.1 & 8.2 \\
\hline Australie & 510 & -6 & 503 & -6 & 494 & -8 & 18.4 & 11.1 \\
\hline Royaume-Uni & 509 & -1 & 498 & 2 & 492 & -1 & 16.9 & 10.1 \\
\hline Allemagne & 509 & -2 & 509 & 6 & 506 & 2 & 19.2 & 9.8 \\
\hline Pays-Bas & 509 & -5 & 503 & -3 & 512 & -6 & 20.0 & 10.9 \\
\hline Suisse & 506 & -2 & 492 & -4 & 521 & -1 & 22.2 & 10.1 \\
\hline Irlande & 503 & 0 & 521 & 13 & 504 & 0 & 15.5 & 6.8 \\
\hline Belgique & 502 & -3 & 499 & -4 & 507 & -5 & 19.7 & 12.7 \\
\hline Danemark & 502 & 2 & 500 & 3 & 511 & -2 & 14.9 & 7.5 \\
\hline Pologne & 501 & 3 & 506 & 3 & 504 & 5 & 15.8 & 8.3 \\
\hline Portugal & 501 & 8 & 498 & 4 & 492 & 7 & 15.6 & 10.7 \\
\hline Norvège & 498 & 3 & 513 & 5 & 502 & 1 & 17.6 & 8.9 \\
\hline Etats-Unis & 496 & 2 & 497 & -1 & 470 & -2 & 13.3 & 13.6 \\
\hline Autriche & 495 & -5 & 485 & -5 & 497 & -2 & 16.2 & 13.5 \\
\hline France & 495 & 0 & 499 & 2 & 493 & -4 & 18.4 & 14.8 \\
\hline Suède & 493 & -4 & 500 & 1 & 494 & -5 & 16.7 & 11.4 \\
\hline République tchèque & 493 & -5 & 487 & 5 & 492 & -6 & 14.0 & 13.7 \\
\hline Espagne & 493 & 2 & 496 & 7 & 486 & 1 & 10.9 & 10.3 \\
\hline Lettonie & 490 & 1 & 488 & 2 & 482 & 0 & 8.3 & 10.5 \\
\hline Russie & 487 & 3 & 495 & 17 & 494 & 6 & 13.0 & 7.7 \\
\hline Luxembourg & 483 & 0 & 481 & 5 & 486 & -2 & 14.1 & 17.0 \\
\hline Italie & 481 & 2 & 485 & 0 & 490 & 7 & 13.5 & 12.2 \\
\hline Hongrie & 477 & -9 & 470 & -12 & 477 & -4 & 10.3 & 18.5 \\
\hline Lituanie & 475 & -3 & 472 & 2 & 478 & -2 & 9.5 & 15.3 \\
\hline Croatie & 475 & -5 & 487 & 5 & 464 & 0 & 9.3 & 14.5 \\
\hline CABA (Argentine) & 475 & 51 & 475 & 46 & 456 & 38 & 7.5 & 14.5 \\
\hline Islande & 473 & -7 & 482 & -9 & 488 & -7 & 13.2 & 13.2 \\
\hline Israël & 467 & 5 & 479 & 2 & 470 & 10 & 13.9 & 20.2 \\
\hline Malte & 465 & 2 & 447 & 3 & 479 & 9 & 15.3 & 21.9 \\
\hline République slovaque & 461 & -10 & 453 & -12 & 475 & -6 & 9.7 & 20.1 \\
\hline Grèce & 455 & -6 & 467 & -8 & 454 & 1 & 6.8 & 20.7 \\
\hline Chili & 447 & 2 & 459 & 5 & 423 & 4 & 3.3 & 23.3 \\
\hline Bulgarie & 446 & 4 & 432 & 1 & 441 & 9 & 6.9 & 29.6 \\
\hline Emirats arabes unis & 437 & -12 & 434 & -8 & 427 & -7 & 5.8 & 31.3 \\
\hline Uruguay & 435 & 1 & 437 & 5 & 418 & -3 & 3.6 & 30.8 \\
\hline Roumanie & 435 & 6 & 434 & 4 & 444 & 10 & 4.3 & 24.3 \\
\hline Chypret 1 & 433 & -5 & 443 & -6 & 437 & -3 & 5.6 & 26.1 \\
\hline Moldavie & 428 & 9 & 416 & 17 & 420 & 13 & 2.8 & 30.1 \\
\hline Albanie & 427 & 18 & 405 & 10 & 413 & 18 & 2.0 & 31.1 \\
\hline Turquie & 425 & 2 & 428 & -18 & 420 & 2 & 1.6 & 31.2 \\
\hline Trinité-et-Tobago & 425 & 7 & 427 & 5 & 417 & 2 & 4.2 & 32.9 \\
\hline Thaillande & 421 & 2 & 409 & -6 & 415 & 1 & 1.7 & 35.8 \\
\hline Costa Rica & 420 & -7 & 427 & -9 & 400 & -6 & 0.9 & 33.0 \\
\hline Qatar & 418 & 21 & 402 & 15 & 402 & 26 & 3.4 & 42.0 \\
\hline Colombie & 416 & 8 & 425 & 6 & 390 & 5 & 1.2 & 38.2 \\
\hline Mexique & 416 & 2 & 423 & -1 & 408 & 5 & 0.6 & 33.8 \\
\hline Monténégro & 411 & 1 & 427 & 10 & 418 & 6 & 2.5 & 33.0 \\
\hline Géorgie & 411 & 23 & 401 & 16 & 404 & 15 & 2.6 & 36.3 \\
\hline Jordanie & 409 & -5 & 408 & 2 & 380 & -1 & 0.6 & 35.7 \\
\hline Indonésie & 403 & 3 & 397 & -2 & 386 & 4 & 0.8 & 42.3 \\
\hline Brésil & 401 & 3 & 407 & -2 & 377 & 6 & 2.2 & 44.1 \\
\hline Pérou & 397 & 14 & 398 & 14 & 387 & 10 & 0.6 & 46.7 \\
\hline Liban & 386 & $\mathrm{~m}$ & 347 & $\mathrm{~m}$ & 396 & $\mathrm{~m}$ & 2.5 & 50.7 \\
\hline Tunisie & 386 & 0 & 361 & -21 & 367 & 4 & 0.6 & 57.3 \\
\hline ERYM & 384 & $\mathrm{~m}$ & 352 & $\mathrm{~m}$ & 371 & $\mathrm{~m}$ & 1.0 & 52.2 \\
\hline Kosovo & 378 & $\mathrm{~m}$ & 347 & $\mathrm{~m}$ & 362 & $\mathrm{~m}$ & 0.0 & 60.4 \\
\hline Algérie & 376 & $\mathrm{~m}$ & 350 & $\mathrm{~m}$ & 360 & $\mathrm{~m}$ & 0.1 & 61.1 \\
\hline République dominicaine & 332 & $\mathrm{~m}$ & 358 & $\mathrm{~m}$ & 328 & $\mathrm{~m}$ & 0.1 & 70.7 \\
\hline
\end{tabular}

1. Note de la Turquie : Les informations figurant dans ce document qui font référence à " Chypre " concernent la partie méridionale de l'lle. II n'y a pas d'autorité unique représentant à la fois les Chypriotes turcs et grecs sur l'lle. La Turquie reconnaît la République Turque de Chypre Nord (RTCN). Jusqu'à ce qu'une solution durable et équitable soit trouvée dans le cadre des Nations Unies, la Turquie maintiendra sa position sur la " question chypriote".

Note de tous les États de l'Union européenne membres de l'OCDE et de l'Union européenne : La République de Chypre est reconnue par tous les membres des Nations Unies sauf la Turquie. Les informations figurant dans ce document concernent la zone sous le contrôle effectif du gouvernement de la République de Chypre.

Remarques : Les valeurs statistiquement significatives sont indiquées en gras.

L'évolution moyenne est indiquée pour la plus longue période disponible depuis PISA 2006 pour les sciences, PISA 2009 pour la compréhension de l'écrit, et PISA 2003 pour les mathématiques. Les pays et économies sont classés par ordre décroissant de leur score moyen en sciences lors de l'évaluation PISA 2015.

Source : Base de données PISA 2015, tableaux I.2.4a, I.2.6, I.2.7, I.4.4a et I.5.4a. 
À une époque où la culture scientifique est de plus en plus liée à la croissance économique et s'avère nécessaire à la résolution de problèmes sociaux et environnementaux complexes, l'ensemble des citoyens, et pas seulement ceux qui se destinent à une carrière de scientifique ou d'ingénieur, doivent être désireux et capables de faire face à des dilemmes scientifiques.

Pendant la majeure partie du $X X^{e}$ siècle, les programmes scolaires de sciences, notamment dans le deuxième cycle de l'enseignement secondaire, avaient tendance à mettre l'accent sur des compétences fondamentales dans le but de former un petit nombre de scientifiques et d'ingénieurs. Dans ces programmes, les sciences étaient présentées aux élèves sous la forme de faits, lois ou théories scientifiques de base, au détriment des grands cadres théoriques et de la nature évolutive de la "vérité " scientifique. Sur la base de l'aptitude des élèves à maîtriser ces faits et théories, les professionnels de l'éducation avaient tendance à identifier ceux qui pouvaient poursuivre des études scientifiques après leur scolarité obligatoire, plutôt qu'à encourager tous les élèves à s'intéresser aux sciences.

Il est important de promouvoir une image positive et inclusive des sciences. On considère trop souvent les cours de sciences comme le premier obstacle sur le parcours du combattant qui conduit finalement à la profession de scientifique ou d'ingénieur. Évoquer la métaphore du parcours du combattant, c'est passer sous silence les nombreuses voies différentes que de brillants scientifiques ont empruntées pour réaliser leurs ambitions professionnelles, mais c'est aussi donner une image négative de ceux qui ne deviendront pas scientifiques ou ingénieurs. La compréhension de la science et les connaissances scientifiques sont utiles bien au-delà des professions scientifiques et sont, comme l'affirme l'enquête PISA, indispensables pour participer pleinement à la vie d'un monde de plus en plus façonné par la science et la technologie. Dans cette perspective, il faudrait promouvoir les cours de sciences de façon plus positive - peut-être comme un « tremplin » vers de nouveaux centres d'intérêt et de divertissement.

\section{Les parents et les enseignants peuvent remettre} en question les stéréotypes liés aux activités et aux professions scientifiques afin d'encourager les filles et les garçons à réaliser leur potentiel.

Parmi les trois domaines d'évaluation de l'enquête PISA (sciences, mathématiques et compréhension de l'écrit), les sciences sont celui où les écarts de performance entre les filles et les garçons sont les plus faibles. Ce constat indique que les écarts de performance entre garçons et filles ne sont pas dus à des différences d'aptitudes innées, mais plutôt à des facteurs sur lesquels les parents, les enseignants, les responsables politiques et les leaders d'opinion peuvent agir.
La plupart des élèves ayant participé à l'évaluation PISA ont exprimé un large intérêt pour les sujets scientifiques et reconnu le rôle important joué par les sciences dans leur vie. Toutefois, seule une minorité d'élèves ont indiqué participer à des activités scientifiques. Les garçons et les filles, et les élèves issus de milieux favorisés et défavorisés, diffèrent souvent dans leur engagement à l'égard de la science et leurs aspirations à exercer une profession scientifique une fois à l'âge adulte. Or les différences entre les sexes concernant l'engagement des élèves en sciences et leurs aspirations professionnelles semblent davantage liées aux différents domaines dans lesquels les filles et les garçons s'estiment performants et qui les intéressent, plutôt qu'à des différences de performance réelle.

Les stéréotypes à l'égard des scientifiques et des professions scientifiques (l'informatique est un domaine « masculin » et la biologie un domaine "féminin »; les scientifiques réussissent parce qu'ils sont doués et pas parce qu'ils travaillent dur ; les scientifiques sont "fous ") peuvent décourager certains élèves de suivre une voie scientifique. En plus de remettre en question les stéréotypes de genre, les parents et les enseignants peuvent soutenir l'engagement des élèves en sciences en les sensibilisant à l'éventail de possibilités de carrières qui s'offrent à eux à la suite d'une formation en science ou en technologie.

\section{Le moyen le plus immédiat de susciter}

l'intérêt pour les sciences chez les élèves dont l'environnement familial est moins propice pourrait consister à augmenter, dans le cadre scolaire, l'offre dès le plus jeune âge d'un enseignement de qualité en sciences.

L'enquête PISA 2015 montre que, dans la plupart des pays et économies, le profil socio-économique et le statut au regard de l'immigration sont associés à des écarts significatifs de performance entre les élèves. En moyenne, dans les pays de l'OCDE, les élèves défavorisés obtiennent ainsi 88 points de moins en sciences que leurs pairs favorisés. En outre, dans plus de 40 pays et économies, après contrôle de la performance des élèves aux épreuves de sciences, les élèves défavorisés restent significativement moins susceptibles que leurs pairs favorisés de s'imaginer poursuivre une carrière scientifique.

Toutefois, les données PISA indiquent également que la corrélation entre les caractéristiques contextuelles des élèves et leurs résultats scolaires varie considérablement entre les pays. Dans certains pays très performants, cette corrélation est plus faible que la moyenne, ce qui implique que des résultats élevés et une éducation équitable ne s'excluent pas mutuellement. Cette corrélation rappelle la définition de l'équité de PISA : une performance élevée pour les élèves de tous les milieux, plutôt que de faibles variations au niveau de la performance des élèves uniquement. Dans l'enquête PISA 2015, le Canada, le Danemark, l'Estonie, Hong-Kong (Chine) et Macao (Chine) se distinguent à la fois par des niveaux élevés de performance et davantage d'équité dans l'éducation. 
Synthèse des convictions, de l'engagement et de la motivation des élèves en sciences

\begin{tabular}{|c|c|c|c|c|c|c|c|c|c|c|}
\hline & \multicolumn{10}{|c|}{ Pays/économies dont les valeurs sont supérieures à la moyenne de l'OCDE } \\
\hline & \multicolumn{10}{|c|}{ Pays/économies dont les valeurs ne s'écartent pas de la moyenne de l'OCDE dans une mesure statistiquement significative } \\
\hline & \multicolumn{10}{|c|}{ Pays/économies dont les valeurs sont inférieures à la moyenne de l'OCDE } \\
\hline & \multirow[b]{2}{*}{$\begin{array}{c}\text { Score moyen } \\
\text { en sciences }\end{array}$} & \multicolumn{2}{|c|}{$\begin{array}{l}\text { Convictions concernant la nature et } \\
\text { l'origine des connaissances scientifiques }\end{array}$} & Pourcent & d'élèves $\mathrm{e}$ & $\begin{array}{l}\text { geant d } \\
\text { tifique }\end{array}$ & cer une profession & Motiv & n à l'idée d'apprendre en & ciences \\
\hline & & $\begin{array}{l}\text { Indice des } \\
\text { convictions } \\
\text { épistémiques } \\
\text { (valeur accordée } \\
\text { à la démarche } \\
\text { scientifique) }\end{array}$ & $\begin{array}{c}\text { Différence } \\
\text { de score associée } \\
\text { à l'augmentation } \\
\text { d'une unité } \\
\text { de l'indice } \\
\text { des convictions } \\
\text { épistémiques }\end{array}$ & $\begin{array}{l}\text { Tous } \\
\text { les élèves }\end{array}$ & Garçons & Filles & $\begin{array}{c}\text { Probabilité accrue } \\
\text { pour les garçons } \\
\text { d'envisager } \\
\text { d'exercer une } \\
\text { profession } \\
\text { scientifique }\end{array}$ & $\begin{array}{l}\text { Indice du plaisir } \\
\text { d'apprendre } \\
\text { en sciences }\end{array}$ & $\begin{array}{c}\text { Différence } \\
\text { de score associée } \\
\text { à l'augmentation } \\
\text { d'une unité de l'indice } \\
\text { du plaisir d'apprendre } \\
\text { en sciences }\end{array}$ & $\begin{array}{c}\text { Différence } \\
\text { entre les sexes } \\
\text { concernant } \\
\text { le plaisir } \\
\text { d'apprendre } \\
\text { en sciences } \\
\text { (garçons - filles) }\end{array}$ \\
\hline & Score moyen & Indice moyen & Diff. de score & $\%$ & $\%$ & $\%$ & Risque relatif & Indice moyen & Diff. de score & Diff. \\
\hline Moyenne OCDE & 493 & 0.00 & 33 & 24.5 & 25.0 & 23.9 & 1.1 & 0.02 & 25 & 0.13 \\
\hline Singapour & 556 & 0.22 & 34 & 28.0 & 31.8 & 23.9 & 1.3 & 0.59 & 35 & 0.17 \\
\hline Japon & 538 & -0.06 & 34 & 18.0 & 18.5 & 17.5 & 1.1 & -0.33 & 27 & 0.52 \\
\hline Estonie & 534 & 0.01 & 36 & 24.7 & 28.9 & 20.3 & 1.4 & 0.16 & 24 & 0.05 \\
\hline Taipei chinois & 532 & 0.31 & 38 & 20.9 & 25.6 & 16.0 & 1.6 & -0.06 & 28 & 0.39 \\
\hline Finlande & 531 & -0.07 & 38 & 17.0 & 15.4 & 18.7 & 0.8 & -0.07 & 30 & 0.04 \\
\hline Macao (Chine) & 529 & -0.06 & 26 & 20.8 & 22.0 & 19.6 & 1.1 & 0.20 & 21 & 0.16 \\
\hline Canada & 528 & 0.30 & 29 & 33.9 & 31.2 & 36.5 & 0.9 & 0.40 & 26 & 0.15 \\
\hline Viet Nam & 525 & -0.15 & 31 & 19.6 & 21.2 & 18.1 & 1.2 & 0.65 & 14 & 0.06 \\
\hline Hong-Kong (Chine) & 523 & 0.04 & 23 & 23.6 & 22.9 & 24.2 & 0.9 & 0.28 & 20 & 0.26 \\
\hline P-S-J-G (Chine) & 518 & -0.08 & 37 & 16.8 & 17.1 & 16.5 & 1.0 & 0.37 & 28 & 0.14 \\
\hline Corée & 516 & 0.02 & 38 & 19.3 & 21.7 & 16.7 & 1.3 & -0.14 & 31 & 0.32 \\
\hline Nouvelle-Zélande & 513 & 0.22 & 40 & 24.8 & 21.7 & 27.9 & 0.8 & 0.20 & 32 & 0.03 \\
\hline Slovénie & 513 & 0.07 & 33 & 30.8 & 34.6 & 26.8 & 1.3 & -0.36 & 22 & -0.03 \\
\hline Australie & 510 & 0.26 & 39 & 29.2 & 30.3 & 28.2 & 1.1 & 0.12 & 33 & 0.16 \\
\hline Royaume-Uni & 509 & 0.22 & 37 & 29.1 & 28.7 & 29.6 & 1.0 & 0.15 & 30 & 0.18 \\
\hline Allemagne & 509 & -0.16 & 34 & 15.3 & 17.4 & 13.2 & 1.3 & -0.18 & 29 & 0.43 \\
\hline Pays-Bas & 509 & -0.19 & 46 & 16.3 & 16.9 & 15.7 & 1.1 & -0.52 & 30 & 0.25 \\
\hline Suisse & 506 & -0.07 & 34 & 19.5 & 19.8 & 19.1 & 1.0 & -0.02 & 30 & 0.17 \\
\hline Irlande & 503 & 0.21 & 36 & 27.3 & 28.0 & 26.6 & 1.1 & 0.20 & 32 & 0.09 \\
\hline Belgique & 502 & 0.00 & 34 & 24.5 & 25.3 & 23.6 & 1.1 & -0.03 & 28 & 0.20 \\
\hline Danemark & 502 & 0.17 & 32 & 14.8 & 11.8 & 17.7 & 0.7 & 0.12 & 26 & 0.09 \\
\hline Pologne & 501 & -0.08 & 27 & 21.0 & 15.4 & 26.8 & 0.6 & 0.02 & 18 & -0.10 \\
\hline Portugal & 501 & 0.28 & 33 & 27.5 & 26.7 & 28.3 & 0.9 & 0.32 & 23 & 0.08 \\
\hline Norvège & 498 & -0.01 & 35 & 28.6 & 28.9 & 28.4 & 1.0 & 0.12 & 29 & 0.27 \\
\hline États-Unis & 496 & 0.25 & 32 & 38.0 & 33.0 & 43.0 & 0.8 & 0.23 & 26 & 0.21 \\
\hline Autriche & 495 & -0.14 & 36 & 22.3 & 26.6 & 18.0 & 1.5 & -0.32 & 25 & 0.23 \\
\hline France & 495 & 0.01 & 30 & 21.2 & 23.6 & 18.7 & 1.3 & -0.03 & 30 & 0.31 \\
\hline Suède & 493 & 0.14 & 38 & 20.2 & 21.8 & 18.5 & 1.2 & 0.08 & 27 & 0.22 \\
\hline République tchèque & 493 & -0.23 & 41 & 16.9 & 18.6 & 15.0 & 1.2 & -0.34 & 27 & -0.06 \\
\hline Espagne & 493 & 0.11 & 30 & 28.6 & 29.5 & 27.8 & 1.1 & 0.03 & 28 & 0.11 \\
\hline Lettonie & 490 & -0.26 & 27 & 21.3 & 21.1 & 21.5 & 1.0 & 0.09 & 18 & 0.03 \\
\hline Russie & 487 & -0.26 & 27 & 23.5 & 23.2 & 23.8 & 1.0 & 0.00 & 16 & 0.07 \\
\hline Luxembourg & 483 & -0.15 & 35 & 21.1 & 24.3 & 18.0 & 1.4 & 0.10 & 26 & 0.14 \\
\hline Italie & 481 & -0.10 & 34 & 22.6 & 24.7 & 20.6 & 1.2 & 0.00 & 22 & 0.24 \\
\hline Hongrie & 477 & -0.36 & 35 & 18.3 & 23.9 & 12.8 & 1.9 & -0.23 & 20 & -0.02 \\
\hline Lituanie & 475 & 0.11 & 22 & 23.9 & 22.5 & 25.4 & 0.9 & 0.36 & 20 & -0.14 \\
\hline Croatie & 475 & 0.03 & 32 & 24.2 & 26.8 & 21.8 & 1.2 & -0.11 & 22 & 0.05 \\
\hline CABA (Argentine) & 475 & 0.09 & 28 & 27.8 & 26.2 & 29.3 & 0.9 & -0.20 & 15 & -0.14 \\
\hline Islande & 473 & 0.29 & 28 & 23.8 & 20.1 & 27.3 & 0.7 & 0.15 & 24 & 0.26 \\
\hline Israël & 467 & 0.18 & 38 & 27.8 & 26.1 & 29.5 & 0.9 & 0.09 & 20 & 0.06 \\
\hline Malte & 465 & 0.09 & 54 & 25.4 & 30.2 & 20.4 & 1.5 & 0.18 & 48 & 0.11 \\
\hline République slovaque & 461 & -0.35 & 36 & 18.8 & 18.5 & 19.0 & 1.0 & -0.24 & 25 & -0.02 \\
\hline Grèce & 455 & -0.19 & 36 & 25.3 & 25.7 & 24.9 & 1.0 & 0.13 & 27 & 0.12 \\
\hline Chili & 447 & -0.15 & 23 & 37.9 & 36.9 & 39.0 & 0.9 & 0.08 & 15 & -0.09 \\
\hline Bulgarie & 446 & -0.18 & 34 & 27.5 & 28.8 & 25.9 & 1.1 & 0.28 & 17 & -0.16 \\
\hline Emirats arabes unis & 437 & 0.04 & 33 & 41.3 & 39.9 & 42.6 & 0.9 & 0.47 & 22 & -0.02 \\
\hline Uruguay & 435 & -0.13 & 27 & 28.1 & 23.8 & 31.9 & 0.7 & -0.10 & 16 & -0.07 \\
\hline Roumanie & 435 & -0.38 & 27 & 23.1 & 23.3 & 23.0 & 1.0 & -0.03 & 17 & -0.05 \\
\hline Chypre $^{1}$ & 433 & -0.15 & 33 & 29.9 & 29.3 & 30.5 & 1.0 & 0.15 & 29 & 0.06 \\
\hline Moldavie & 428 & -0.14 & 37 & 22.0 & 22.5 & 21.3 & 1.1 & 0.33 & 22 & -0.17 \\
\hline Albanie & 427 & -0.03 & $\mathrm{~m}$ & 24.8 & $\mathrm{~m}$ & $\mathrm{~m}$ & $\mathrm{~m}$ & 0.72 & $\mathrm{~m}$ & $\mathrm{~m}$ \\
\hline Turquie & 425 & -0.17 & 18 & 29.7 & 34.5 & 24.9 & 1.4 & 0.15 & 12 & 0.01 \\
\hline Trinité-et-Tobago & 425 & -0.02 & 28 & 27.8 & 24.6 & 31.0 & 0.8 & 0.19 & 24 & -0.01 \\
\hline Thaillande & 421 & -0.07 & 35 & 19.7 & 12.4 & 25.2 & 0.5 & 0.42 & 18 & -0.05 \\
\hline Costa Rica & 420 & -0.15 & 16 & 44.0 & 43.8 & 44.2 & 1.0 & 0.35 & 4 & -0.03 \\
\hline Qatar & 418 & -0.10 & 33 & 38.0 & 36.3 & 39.9 & 0.9 & 0.36 & 25 & 0.00 \\
\hline Colombie & 416 & -0.19 & 21 & 39.7 & 37.1 & 42.0 & 0.9 & 0.32 & 7 & -0.02 \\
\hline Mexique & 416 & -0.17 & 17 & 40.7 & 45.4 & 35.8 & 1.3 & 0.42 & 12 & 0.01 \\
\hline Monténégro & 411 & -0.32 & 23 & 21.2 & 20.1 & 22.4 & 0.9 & 0.09 & 14 & -0.07 \\
\hline Géorgie & 411 & 0.05 & 42 & 17.0 & 16.4 & 17.7 & 0.9 & 0.34 & 23 & -0.13 \\
\hline Jordanie & 409 & -0.13 & 28 & 43.7 & 44.6 & 42.8 & 1.0 & 0.53 & 23 & -0.25 \\
\hline Indonésie & 403 & -0.30 & 16 & 15.3 & 8.6 & 22.1 & 0.4 & 0.65 & 6 & -0.06 \\
\hline Brésil & 401 & -0.07 & 27 & 38.8 & 34.4 & 42.8 & 0.8 & 0.23 & 19 & -0.04 \\
\hline Pérou & 397 & -0.16 & 23 & 38.7 & 42.7 & 34.6 & 1.2 & 0.40 & 9 & 0.01 \\
\hline Liban & 386 & -0.24 & 35 & 39.7 & 41.0 & 38.5 & 1.1 & 0.38 & 32 & -0.04 \\
\hline Tunisie & 386 & -0.31 & 18 & 34.4 & 28.5 & 39.5 & 0.7 & 0.52 & 15 & -0.12 \\
\hline ERYM & 384 & -0.18 & 30 & 24.2 & 20.0 & 28.8 & 0.7 & 0.48 & 17 & -0.29 \\
\hline Kosovo & 378 & 0.03 & 22 & 26.4 & 24.7 & 28.1 & 0.9 & 0.92 & 14 & -0.16 \\
\hline Algérie & 376 & -0.31 & 16 & 26.0 & 23.1 & 29.2 & 0.8 & 0.46 & 14 & -0.12 \\
\hline République dominicaine & 332 & -0.10 & 13 & 45.7 & 44.7 & 46.8 & 1.0 & 0.54 & 6 & -0.05 \\
\hline
\end{tabular}

1. Note de la Turquie : Les informations figurant dans ce document qui font référence à « Chypre " concernent la partie méridionale de l'lle. II n'y a pas d'autorité unique représentant à la fois les Chypriotes turcs et grecs sur l'lle. La Turquie reconnaît la République Turque de Chypre Nord (RTCN). Jusqu'à ce qu'une solution durable et équitable soit trouvée dans le cadre des Nations Unies, la Turquie maintiendra sa position sur la " question chypriote".

Note de tous les États de l'Union européenne membres de l'OCDE et de l'Union européenne : La République de Chypre est reconnue par tous les membres des Nations Unies sauf la Turquie. Les informations figurant dans ce document concernent la zone sous le contrôle effectif du gouvernement de la République de Chypre.

Remarque : Les valeurs statistiquement significatives sont indiquées en gras.

Les pays et économies sont classés par ordre décroissant de leur score moyen en sciences lors de l'évaluation PISA 2015.

Source : OCDE, Base de données PISA 2015, tableaux I.2.12a-b, I.3.1a-c et I.3.10a-b. 
Pour les élèves défavorisés et ceux qui ont des difficultés en sciences, des ressources supplémentaires, qui ciblent soit les élèves au niveau individuel, soit les établissements défavorisés, peuvent faire la différence en aidant les élèves à atteindre le seuil de compétence en sciences et à développer leur intérêt en la matière tout au long de la vie. Tous les élèves, qu'ils soient issus de l'immigration ou non, qu'ils soient favorisés ou défavorisés, bénéficieraient également de politiques publiques moins sélectives, en particulier lorsque ces politiques qui visent à répartir les élèves dans différents programmes d'enseignement ou différents établissements sont appliquées durant les premières années de l'enseignement secondaire. Ces politiques contribuent souvent à l'apparition de disparités dans l'enseignement des sciences - tant en termes de quantité que de qualité - qui est proposé aux élèves issus de différents milieux. II pourra être nécessaire de développer des programmes spécifiques en vue d'éveiller l'intérêt pour les sciences chez les élèves qui ne trouvent peut-être pas cette motivation en dehors du cadre scolaire, et de soutenir les élèves dans leur décision de poursuivre des études scientifiques. Offrir aux élèves davantage de possibilités d'étudier les sciences les aidera à apprendre à " réfléchir comme des scientifiques ", une compétence devenue essentielle au XXle siècle, que les élèves choisissent d'exercer une profession scientifique ou non.
1. Par indice SESC, on entend l'indice PISA de statut économique, social et culturel. 2. Toutes les différences de score en sciences associées à l'augmentation d'une unité de l'indice PISA de statut économique, social et culturel sont statistiquement significatives.

3. Par élèves résilients, on entend les élèves qui se situent dans le quartile inférieur de l'indice PISA de statut économique, social et culturel (SESC) d'un pays ou d'une économie, et qui se classent dans le quartile supérieur de la performance tous pays et économies confondus, après contrôle du niveau socio-économique.

4. Une valeur positive indique une différence de score en faveur des élèves autochtones; une valeur négative, une différence de score en faveur des élèves issus de l'immigration.

5. Note de la Turquie : Les informations figurant dans ce document qui font référence à "Chypre * concernent la partie méridionale de l'lle. II n'y a pas d'autorité unique représentant à la fois les Chypriotes turcs et grecs sur l'lle. La Turquie reconnaît la République Turque de Chypre Nord (RTCN) Jusqu'à ce qu'une solution durable et équitable soit trouvée dans le cadre des Nations Unies, la Turquie maintiendra sa position sur la « question chypriote".

Note de tous les États de l'Union européenne membres de l'OCDE et de l'Union européenne : La République de Chypre est reconnue par tous les membres des Nations Unies sauf la Turquie. Les informations figurant dans ce document concernent la zone sous le contrôle effectif du gouvernement de la République de Chypre.

Remarques : Les valeurs statistiquement significatives sont indiquées en gras.

Les pays et économies sont classés par ordre décroissant de leur score moyen en sciences lors de l'évaluation PISA 2015.

Source : OCDE, Base de données PISA 2015, tableaux I.2.3, I.6.1, I.6.3a, I.6.7, I.6.17, I.7.1 et I.7.15a

\begin{tabular}{|c|c|c|c|}
\hline & $\begin{array}{l}\text { Score moyen en } \\
\text { sciences } \\
\text { lors de l'évaluation } \\
\text { PISA 2015 }\end{array}$ & $\begin{array}{c}\text { Couverture de } \\
\text { la population nationale } \\
\text { de jeunes de } 15 \text { ans } \\
\text { (indice PSA de couverture 3) }\end{array}$ & $\begin{array}{l}\text { Pourcentage de la variation } \\
\text { de la performance } \\
\text { en sciences expliqué } \\
\text { par le niveau } \\
\text { socio-économique } \\
\text { des élèves }\end{array}$ \\
\hline & Score moyen & Indice moyen & $\%$ \\
\hline Moyenne OCDE & 493 & 0.89 & 12.9 \\
\hline Singapour & 556 & 0.96 & 17 \\
\hline Japon & 538 & 0.95 & 10 \\
\hline Estonie & 534 & 0.93 & 8 \\
\hline Taipei chinois & 532 & 0.85 & 14 \\
\hline Finlande & 531 & 0.97 & 10 \\
\hline Macao (Chine) & 529 & 0.88 & 2 \\
\hline Canada & 528 & 0.84 & 9 \\
\hline Viet Nam & 525 & 0.49 & 11 \\
\hline Hong-Kong (Chine) & 523 & 0.89 & 5 \\
\hline P-S-J-G (Chine) & 518 & 0.64 & 18 \\
\hline Corée & 516 & 0.92 & 10 \\
\hline Nouvelle-Zélande & 513 & 0.90 & 14 \\
\hline Slovénie & 513 & 0.93 & 13 \\
\hline Australie & 510 & 0.91 & 12 \\
\hline Royaume-Uni & 509 & 0.84 & 11 \\
\hline Allemagne & 509 & 0.96 & 16 \\
\hline Pays-Bas & 509 & 0.95 & 13 \\
\hline Suisse & 506 & 0.96 & 16 \\
\hline Irlande & 503 & 0.96 & 13 \\
\hline Belgique & 502 & 0.93 & 19 \\
\hline Danemark & 502 & 0.89 & 10 \\
\hline Pologne & 501 & 0.91 & 13 \\
\hline Portugal & 501 & 0.88 & 15 \\
\hline Norvège & 498 & 0.91 & 8 \\
\hline États-Unis & 496 & 0.84 & 11 \\
\hline Autriche & 495 & 0.83 & 16 \\
\hline France & 495 & 0.91 & 20 \\
\hline Suède & 493 & 0.94 & 12 \\
\hline République tchèque & 493 & 0.94 & 19 \\
\hline Espagne & 493 & 0.91 & 13 \\
\hline Lettonie & 490 & 0.89 & 9 \\
\hline Russie & 487 & 0.95 & 7 \\
\hline Luxembourg & 483 & 0.88 & 21 \\
\hline Italie & 481 & 0.80 & 10 \\
\hline Hongrie & 477 & 0.90 & 21 \\
\hline Lituanie & 475 & 0.90 & 12 \\
\hline Croatie & 475 & 0.91 & 12 \\
\hline CABA (Argentine) & 475 & 1.04 & 26 \\
\hline Islande & 473 & 0.93 & 5 \\
\hline Israël & 467 & 0.94 & 11 \\
\hline Malte & 465 & 0.98 & 14 \\
\hline République slovaque & 461 & 0.89 & 16 \\
\hline Grèce & 455 & 0.91 & 13 \\
\hline Chili & 447 & 0.80 & 17 \\
\hline Bulgarie & 446 & 0.81 & 16 \\
\hline Émirats arabes unis & 437 & 0.91 & 5 \\
\hline Uruguay & 435 & 0.72 & 16 \\
\hline Roumanie & 435 & 0.93 & 14 \\
\hline Chypre $^{5}$ & 433 & 0.95 & 9 \\
\hline Moldavie & 428 & 0.93 & 12 \\
\hline Albanie & 427 & 0.84 & $\mathrm{~m}$ \\
\hline Turquie & 425 & 0.70 & 9 \\
\hline Trinité-et-Tobago & 425 & 0.76 & 10 \\
\hline Thaillande & 421 & 0.71 & 9 \\
\hline Costa Rica & 420 & 0.63 & 16 \\
\hline Qatar & 418 & 0.93 & 4 \\
\hline Colombie & 416 & 0.75 & 14 \\
\hline Mexique & 416 & 0.62 & 11 \\
\hline Monténégro & 411 & 0.90 & 5 \\
\hline Géorgie & 411 & 0.79 & 11 \\
\hline Jordanie & 409 & 0.86 & 9 \\
\hline Indonésie & 403 & 0.68 & 13 \\
\hline Brésil & 401 & 0.71 & 12 \\
\hline Pérou & 397 & 0.74 & 22 \\
\hline Liban & 386 & 0.66 & 10 \\
\hline Tunisie & 386 & 0.93 & 9 \\
\hline ERYM & 384 & 0.95 & 7 \\
\hline Kosovo & 378 & 0.71 & 5 \\
\hline Algérie & 376 & 0.79 & 1 \\
\hline République dominicaine & 332 & 0.68 & 13 \\
\hline
\end{tabular}


Pays/économies dont la performance ou le niveau d'équité sont supérieurs à la moyenne de l'OCDE

Pays/économies dont les valeurs ne s'écartent pas de la moyenne de l'OCDE dans une mesure statistiquement significative

Pays/économies dont la performance ou le niveau d'équité sont inférieurs à la moyenne de l'OCDE

\begin{tabular}{|c|c|c|c|c|c|c|}
\hline \multicolumn{3}{|l|}{ Indicateurs d'inclusion et d'égalité } & \multicolumn{4}{|c|}{ Différence entre PISA 2006 et PISA 2015 (PISA 2015 - PISA 2006) } \\
\hline $\begin{array}{c}\text { Différence de score } \\
\text { en sciences associée } \\
\text { à l'augmentation d'une unité } \\
\text { de l'indice SESC' }\end{array}$ & $\begin{array}{l}\text { Pourcentage d'élèves } \\
\text { résilients }^{3}\end{array}$ & $\begin{array}{l}\text { Différence de performance } \\
\text { en sciences entre les élèves } \\
\text { issus de l'immigration } \\
\text { et les élèves autochtones, } \\
\text { après contrôle de l'indice } \\
\text { SESC et de la langue } \\
\text { parlée en famille }\end{array}$ & $\begin{array}{c}\text { Pourcentage } \\
\text { de la variation } \\
\text { de la performance en sciences } \\
\text { expliqué par le niveau } \\
\text { socio-économique } \\
\text { des élèves }\end{array}$ & $\begin{array}{c}\text { Différence de score } \\
\text { en sciences associée } \\
\text { à l'augmentation d'une unité } \\
\text { de l'indice SESC }\end{array}$ & $\begin{array}{l}\text { Pourcentage d'élèves } \\
\text { résilients }\end{array}$ & $\begin{array}{c}\text { Différence de performance } \\
\text { en sciences entre les élèves } \\
\text { issus de l'immigration } \\
\text { et les élèves autochtones, } \\
\text { après contrôle de l'indice } \\
\text { SESC et de la langue } \\
\text { parlée en famille }\end{array}$ \\
\hline Diff. de score ${ }^{2}$ & $\%$ & Diff. de score & Diff. de \% & Diff. de score & Diff. de \% & Diff. de score \\
\hline 38 & 29.2 & 19 & -1.4 & 0 & 1.5 & -6 \\
\hline 47 & 48.8 & -13 & $\mathrm{~m}$ & $\mathrm{~m}$ & $\mathrm{~m}$ & $\mathrm{~m}$ \\
\hline 42 & 48.8 & 53 & 1.6 & 2 & 8.2 & $\mathrm{~m}$ \\
\hline 32 & 48.3 & 28 & -1.0 & 2 & 2.0 & -2 \\
\hline 45 & 46.3 & $\mathrm{~m}$ & 1.0 & 2 & 2.0 & $\mathrm{~m}$ \\
\hline 40 & 42.8 & 36 & 1.8 & 10 & -10.4 & -11 \\
\hline 12 & 64.6 & -19 & -0.1 & 0 & 5.8 & -2 \\
\hline 34 & 38.7 & -5 & 0.3 & 1 & 0.7 & -11 \\
\hline 23 & 75.5 & $\mathrm{~m}$ & $\mathrm{~m}$ & $\mathrm{~m}$ & $\mathrm{~m}$ & $\mathrm{~m}$ \\
\hline 19 & 61.8 & -1 & -1.5 & -8 & -0.7 & 10 \\
\hline 40 & 45.3 & 135 & $\mathrm{~m}$ & $\mathrm{~m}$ & $\mathrm{~m}$ & $\mathrm{~m}$ \\
\hline 44 & 40.4 & $\mathrm{~m}$ & 3.1 & 13 & -3.2 & $\mathrm{~m}$ \\
\hline 49 & 30.4 & -3 & -2.0 & 0 & -4.7 & -9 \\
\hline 43 & 34.6 & 14 & -4.0 & -5 & 4.3 & 1 \\
\hline 44 & 32.9 & $\begin{array}{l}-13 \\
\end{array}$ & -0.4 & 2 & -0.2 & -8 \\
\hline 37 & 35.4 & 15 & -2.9 & -8 & 5.0 & 9 \\
\hline 42 & 33.5 & 28 & -4.0 & -5 & 8.7 & 7 \\
\hline 47 & 30.7 & 23 & -3.8 & 3 & -1.3 & $\begin{array}{l}-10 \\
\end{array}$ \\
\hline 43 & 29.1 & 16 & -0.7 & 0 & 1.2 & -20 \\
\hline 38 & 29.6 & 3 & -0.5 & 1 & 0.4 & 6 \\
\hline 48 & 27.2 & 28 & -0.7 & 2 & 1.4 & -32 \\
\hline 34 & 27.5 & 38 & -3.6 & -7 & 7.9 & 7 \\
\hline 40 & 34.6 & $\mathrm{~m}$ & -1.4 & 0 & 3.2 & $\mathrm{~m}$ \\
\hline 31 & 38.1 & 8 & -1.4 & 3 & 4.4 & -49 \\
\hline 37 & 26.5 & 23 & -0.4 & 1 & 9.3 & 8 \\
\hline 33 & 31.6 & -5 & -6.0 & -13 & 12.3 & -10 \\
\hline 45 & 25.9 & 18 & 0.1 & 0 & -2.2 & -17 \\
\hline 57 & 26.6 & 20 & -1.9 & 5 & 3.0 & 10 \\
\hline 44 & 24.7 & 40 & 1.2 & 6 & 0.6 & 13 \\
\hline 52 & 24.9 & 2 & 2.7 & 1 & -3.9 & -20 \\
\hline 27 & 39.2 & 26 & 0.9 & 3 & 10.7 & -23 \\
\hline 26 & 35.2 & 14 & -0.5 & -4 & 6.0 & 7 \\
\hline 29 & 25.5 & 5 & -0.9 & 0 & -1.0 & -4 \\
\hline 41 & 20.7 & 22 & -1.7 & 2 & 1.5 & -16 \\
\hline 30 & 26.6 & 11 & -0.6 & -1 & 2.8 & -32 \\
\hline 47 & 19.3 & -11 & 0.3 & 2 & -6.7 & -13 \\
\hline 36 & 23.1 & 2 & -2.6 & -2 & -2.1 & 11 \\
\hline 38 & 24.4 & 14 & -0.1 & 3 & -0.5 & 7 \\
\hline 37 & 14.9 & 15 & $\mathrm{~m}$ & $\mathrm{~m}$ & $\mathrm{~m}$ & $\mathrm{~m}$ \\
\hline 28 & 17.0 & 53 & -2.6 & -3 & -1.8 & 24 \\
\hline 42 & 15.7 & -9 & 0.9 & 0 & 2.3 & 1 \\
\hline 47 & 21.8 & -5 & $\mathrm{~m}$ & $\mathrm{~m}$ & $\mathrm{~m}$ & $\mathrm{~m}$ \\
\hline 41 & 17.5 & 40 & -3.6 & -4 & -2.8 & $\mathrm{~m}$ \\
\hline 34 & 18.1 & 14 & -2.1 & -2 & -2.3 & 5 \\
\hline 32 & 14.6 & 21 & -6.4 & -6 & -0.4 & $\mathrm{~m}$ \\
\hline 41 & 13.6 & 49 & -6.3 & $\begin{array}{l}-7 \\
\end{array}$ & 4.1 & $m$ \\
\hline 30 & 7.7 & -77 & $\mathrm{~m}$ & $\mathrm{~m}$ & $\mathrm{~m}$ & $\mathrm{~m}$ \\
\hline 32 & 14.0 & 11 & -1.6 & -2 & -1.8 & $\mathrm{~m}$ \\
\hline 34 & $\begin{array}{l}11.3 \\
\end{array}$ & $\mathrm{~m}$ & $\begin{array}{l}1.5 \\
\end{array}$ & $\begin{array}{l}-1 \\
\end{array}$ & 4.8 & $\mathrm{~m}$ \\
\hline 31 & 10.1 & 1 & $\mathrm{~m}$ & $\mathrm{~m}$ & $\mathrm{~m}$ & $\mathrm{~m}$ \\
\hline 33 & 13.4 & 0 & $\mathrm{~m}$ & $\mathrm{~m}$ & $\mathrm{~m}$ & $\mathrm{~m}$ \\
\hline $\mathrm{m}$ & $\mathrm{m}$ & $\mathrm{m}$ & $\mathrm{m}$ & $\mathrm{m}$ & $\mathrm{m}$ & $\mathrm{m}$ \\
\hline 20 & 21.8 & 22 & -6.1 & -7 & -1.4 & 21 \\
\hline 31 & 12.9 & 19 & $\mathrm{~m}$ & $\mathrm{~m}$ & $\mathrm{~m}$ & $\mathrm{~m}$ \\
\hline 22 & 18.4 & -8 & -6.5 & -5 & -5.2 & $\mathrm{~m}$ \\
\hline 24 & 9.4 & 6 & $\mathrm{~m}$ & $\mathrm{~m}$ & $\mathrm{~m}$ & $\mathrm{~m}$ \\
\hline 27 & 5.7 & -77 & 2.4 & 15 & 4.9 & -19 \\
\hline 27 & 11.4 & 60 & 3.1 & 4 & 0.3 & $\mathrm{~m}$ \\
\hline 19 & 12.8 & 57 & -5.2 & -5 & -1.9 & -21 \\
\hline 23 & 9.4 & -7 & -2.6 & -1 & 1.8 & 12 \\
\hline 34 & 7.5 & 4 & $\mathrm{~m}$ & $\mathrm{~m}$ & $\mathrm{~m}$ & $\mathrm{~m}$ \\
\hline 25 & 7.7 & -2 & -1.6 & 0 & -6.6 & 13 \\
\hline 22 & 10.9 & $\mathrm{~m}$ & 3.5 & 1 & -4.1 & $\mathrm{~m}$ \\
\hline 27 & 9.4 & 64 & -4.5 & $\begin{array}{l}-1 \\
\end{array}$ & -0.9 & 30 \\
\hline 30 & 3.2 & 29 & $\mathrm{~m}$ & $\mathrm{~m}$ & $\mathrm{~m}$ & $\mathrm{~m}$ \\
\hline 26 & 6.1 & 18 & $\mathrm{~m}$ & $\mathrm{~m}$ & $\mathrm{~m}$ & $\mathrm{~m}$ \\
\hline 17 & 4.7 & 50 & 0.1 & -2 & -11.7 & -20 \\
\hline 25 & 4.1 & 23 & $\mathrm{~m}$ & $\mathrm{~m}$ & $\mathrm{~m}$ & $\mathrm{~m}$ \\
\hline 18 & 2.5 & 28 & $\mathrm{~m}$ & $\mathrm{~m}$ & $\mathrm{~m}$ & $m$ \\
\hline 8 & 7.4 & 33 & $\mathrm{~m}$ & $\mathrm{~m}$ & $\mathrm{~m}$ & $\mathrm{~m}$ \\
\hline 25 & 0.4 & 26 & $\mathrm{~m}$ & $\mathrm{~m}$ & $\mathrm{~m}$ & $\mathrm{~m}$ \\
\hline
\end{tabular}




\section{Politiques et pratiques pour des établissements performants}

\section{Que nous apprennent les résultats?}

\section{Politiques relatives à l'apprentissage des sciences à l'école et performance des élèves en sciences}

- Dans les pays de l'OCDE, les élèves déclarant ne pas suivre de cours de sciences (soit environ $6 \%$ des effectifs) obtiennent un score en sciences inférieur de 25 points à celui des élèves déclarant suivre au moins un cours de sciences par semaine, après contrôle du profil socio-économique des élèves et des établissements. Dans 34 systèmes d'éducation, notamment en Allemagne, en Autriche, en Belgique, en Croatie, en France, en République slovaque et au Taipei chinois, les élèves déclarant ne pas suivre de cours de sciences sont plus susceptibles d'être scolarisés dans des établissements défavorisés sur le plan socio-économique.

- Dans les pays de l'OCDE, les établissements favorisés sur le plan socio-économique sont bien plus susceptibles que les établissements défavorisés de proposer des compétitions de sciences et un club de sciences dans le cadre de leurs activités scolaires.

- La performance en sciences des élèves et leur aspiration à exercer une profession scientifique sont davantage corrélées au temps consacré à l'apprentissage des sciences et à la manière dont ces disciplines sont enseignées qu'à des facteurs comme le matériel et le personnel affectés à la section des sciences, la nature des activités scientifiques extrascolaires proposées dans les établissements, ou encore les qualifications des professeurs de sciences.

- En moyenne, dans les pays de l'OCDE, selon les déclarations des élèves, les enseignants des établissements favorisés expliquent ou démontrent plus fréquemment des concepts scientifiques (enseignement dirigé par l'enseignant) que leurs homologues des établissements défavorisés. Les élèves indiquant que leurs professeurs de sciences ont souvent recours à ces méthodes et adaptent leur enseignement pour répondre aux besoins des élèves obtiennent un meilleur score en sciences, accordent davantage de valeur à la démarche scientifique et sont plus susceptibles d'envisager d'exercer une profession scientifique, par rapport aux élèves dont les professeurs utilisent ces méthodes moins fréquemment.

\section{Environnements d'apprentissage}

- Dans la plupart des systèmes d'éducation, les élèves scolarisés dans un établissement défavorisé sur le plan socio-économique sont plus susceptibles d'avoir déjà séché une journée de cours que ceux scolarisés dans un établissement favorisé. Entre 2012 et 2015, le pourcentage d'élèves ayant séché une journée entière de cours au moins une fois durant les deux semaines précédant l'évaluation PISA a augmenté d'environ 5 points de pourcentage dans les pays de l'OCDE.
- Dans les pays de l'OCDE, selon les chefs d'établissement, l'absentéisme des élèves et la résistance au changement de la part du personnel représentent les facteurs qui entravent le plus l'apprentissage des élèves. En revanche, la consommation d'alcool ou de substances illicites, et les comportements menaçants ou brutaux envers d'autres élèves, constituent les facteurs qui entravent le moins l'apprentissage des élèves.

- Les élèves scolarisés dans un système qui pratique la sélection dans différents types d'établissement ou de filières d'enseignement à un âge plus avancé indiquent bénéficier d'un plus grand soutien de la part de leurs enseignants.

\section{Gouvernance des établissements, évaluations et responsabilisation}

- Les élèves des établissements privés obtiennent un score plus élevé en sciences que ceux des établissements publics. Cependant, après contrôle du profil socio-économique des élèves et des établissements, les élèves des établissements publics obtiennent un score plus élevé que les élèves des établissements privés, en moyenne, dans les pays de l'OCDE et dans 22 systèmes d'éducation.

- Les tests normalisés sont largement utilisés dans les pays et économies participant à l'enquête PISA. Dans environ cinq systèmes d'éducation sur six, au moins la moitié des élèves sont évalués au moins une fois par an à l'aide de tests normalisés obligatoires, et dans environ trois pays sur quatre, au moins la moitié des élèves sont évalués au moins une fois par an à l'aide de tests normalisés facultatifs.

- Lors du choix de l'établissement de leur enfant, les parents sont plus susceptibles de considérer comme critère important, voire très important, sa sécurité, sa réputation, et son ambiance dynamique et agréable - davantage encore que les résultats scolaires de ses élèves.

\section{Sélection et regroupement des élèves}

- Dans 30 pays et économies, le recours au redoublement est moindre en 2015 qu'en 2009, et seuls 5 pays l'ont utilisé davantage durant la période à l'étude. Le recours au redoublement enregistre une diminution d'au moins 10 points de pourcentage au Costa Rica, en France, en Indonésie, en Lettonie, à Macao (Chine), à Malte, au Mexique et en Tunisie.

- Dans les pays de l'OCDE, les garçons et les élèves issus de l'immigration ou de milieux socio-économiques défavorisés sont plus susceptibles d'avoir déjà redoublé, même après contrôle de leurs résultats scolaires, ainsi que de la motivation et des attitudes dont ils font part dans leurs déclarations.

- Plus la première sélection des élèves dans différents établissements ou filières d'enseignement s'opère tardivement, et moins 
Différences concernant l'obligation d'assister aux cours normaux de sciences, selon le profil socio-économique des établissements

\section{Résultats fondés sur les déclarations des élèves}

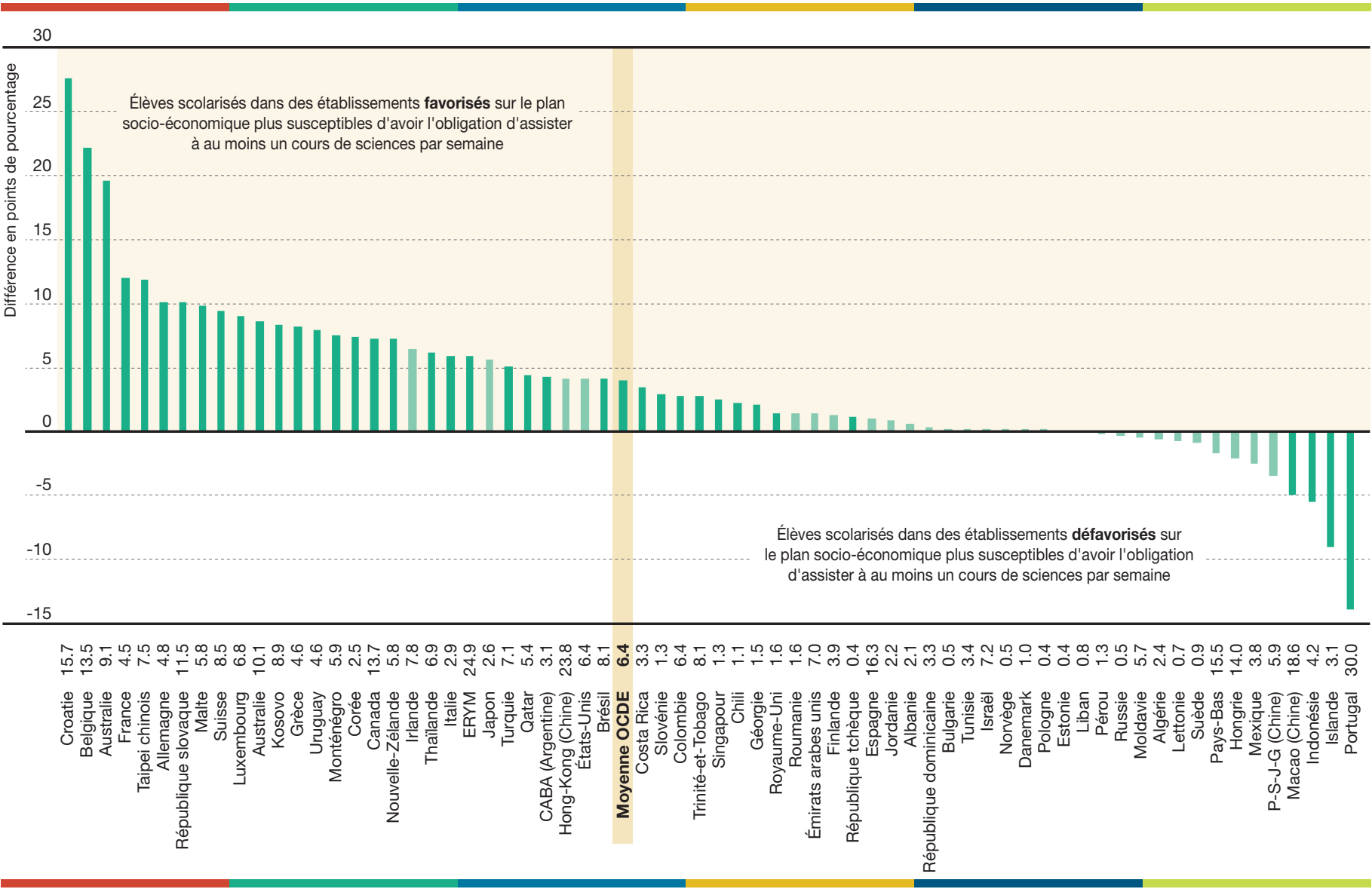

Remarques : Les différences statistiquement significatives sont indiquées dans une couleur plus foncée.

Le pourcentage d'élèves n'ayant l'obligation d'assister à aucun cours de sciences est indiqué en regard du nom du pays/de l'économie.

Les pays et économies sont classés par ordre décroissant de la différence (exprimée en points de pourcentage) d'obligation d'assister à au moins un cours de sciences par semaine entre les élèves scolarisés dans des établissements favorisés sur le plan socio-économique et les élèves scolarisés dans des établissements défavorisés sur le plan socio-économique.

Source : OCDE, Base de données PISA 2015, tableau II.2.3.

les redoublements sont fréquents, plus le système d'éducation est équitable ou plus la corrélation entre le milieu socio-économique des élèves et leur performance en sciences est faible.

\section{Ressources investies dans l'éducation}

- Les élèves des établissements de grande taille obtiennent un meilleur score en sciences et sont plus susceptibles d'envisager une future carrière professionnelle dans le domaine des sciences que les élèves des établissements de plus petite taille. En revanche, les élèves des petits établissements font état d'un meilleur climat de discipline dans leurs cours de sciences et sont moins susceptibles que les élèves des grands établissements de faire preuve d'absentéisme et d'arriver en retard à l'école, après contrôle du profil socio-économique des établissements et des élèves.

- En moyenne, dans les pays de l'OCDE, les élèves des classes à effectif plus réduit indiquent plus fréquemment que les élèves des classes à effectif plus important que leurs enseignants adaptent leur enseignement en fonction de leurs besoins, de leurs connaissances et de leur compréhension.
- Le score des élèves en sciences augmente de 5 points pour toute heure de cours hebdomadaire supplémentaire en sciences, après contrôle du milieu socio-économique.

- Les systèmes d'éducation dans lesquels les élèves consacrent davantage de temps à l'apprentissage en dehors de leurs journées de classe, en faisant leurs devoirs, en suivant des cours supplémentaires ou pour leur travail personnel, tendent à obtenir de moins bons résultats en sciences.

\section{Même si tous les élèves ne sont pas tenus} d'apprendre les mêmes contenus scientifiques, la possibilité de choisir des cours de sciences ne doit pas devenir une possibilité de ne pas apprendre les sciences.

Les élèves qui ne suivent aucun cours de sciences dans le cadre scolaire obtiennent un score en sciences inférieur de 44 points à celui des élèves suivant au moins un cours de sciences par semaine ; 
cet écart représente au moins 50 points dans 21 pays et économies. Leur faible performance peut en partie expliquer pourquoi ces élèves ne suivent pas de cours dans cette discipline, mais les priver entièrement d'un apprentissage des sciences dans le cadre scolaire ne fera que creuser davantage l'écart avec les élèves plus performants.

Toutes les analyses corrélationnelles présentées dans l'enquête PISA suggèrent que l'apprentissage des sciences est plus efficace quand il a lieu dans le cadre scolaire qu'après les journées de classe. Concernant l'apprentissage des sciences, les élèves qui y consacrent plus de temps dans le cadre scolaire obtiennent un score plus élevé en sciences, ce qui n'est pas nécessairement le cas des élèves qui y consacrent plus de temps après les journées de classe. Les élèves obtiennent également un score plus élevé en sciences qu'en mathématiques et qu'en compréhension de l'écrit lorsqu'ils consacrent davantage de temps, dans le cadre scolaire, à l'apprentissage des sciences qu'à celui de ces deux autres disciplines ; mais cela est moins évident lorsque cet apprentissage a lieu après les journées de classe.

Tandis qu'il est difficile de modifier la manière dont les enseignants font cours, les chefs d'établissement et les pouvoirs publics doivent tenter de trouver des solutions afin de renforcer l'efficacité de l'enseignement.

Ce qui se passe en classe joue un rôle crucial dans l'apprentissage des élèves et la formation de leurs aspirations professionnelles.
La manière dont les professeurs enseignent les sciences est davantage corrélée à la performance des élèves en sciences et à leur aspiration à embrasser une carrière scientifique que ne le sont les ressources matérielles et humaines des sections des sciences, y compris la qualification des enseignants ou le type d'activités scientifiques extrascolaires proposées aux élèves. À titre d'exemple, dans la quasi-totalité des systèmes d'éducation, les élèves obtiennent un score plus élevé en sciences lorsqu'ils indiquent que leurs professeurs de sciences "expliquent des concepts scientifiques ", " discutent de leurs questions » ou " démontrent un concept » plus fréquemment. Dans la quasi-totalité des systèmes d'éducation, la performance des élèves en sciences est également meilleure lorsqu'ils indiquent que leurs professeurs de sciences " adaptent leurs cours aux besoins et aux connaissances de la classe » ou " apportent une aide personnalisée quand un élève a des difficultés à comprendre un sujet ou un exercice ".

L'octroi d'une plus grande autonomie aux établissements en matière de programmes scolaires peut donner davantage la possibilité aux enseignants d'adapter leur enseignement en fonction des besoins et des connaissances de leurs élèves. Les élèves affichent un score plus élevé en sciences dans les systèmes d'éucation où les chefs d'établissement disposent d'une plus grande autonomie en matière de ressources, de programmes scolaires et d'autres politiques scolaires, et plus particulièrement dans les pays où il existe un suivi dans le temps ou une publication des résultats scolaires, et où les chefs d'établissement s'investissent davantage dans la direction pédagogique. Ces constats mettent en avant l'interdépendance entre

Résultats fondés sur les déclarations des élèves ; pourcentage d'élèves ayant séché une journée de cours durant les deux semaines précédant l'évaluation PISA

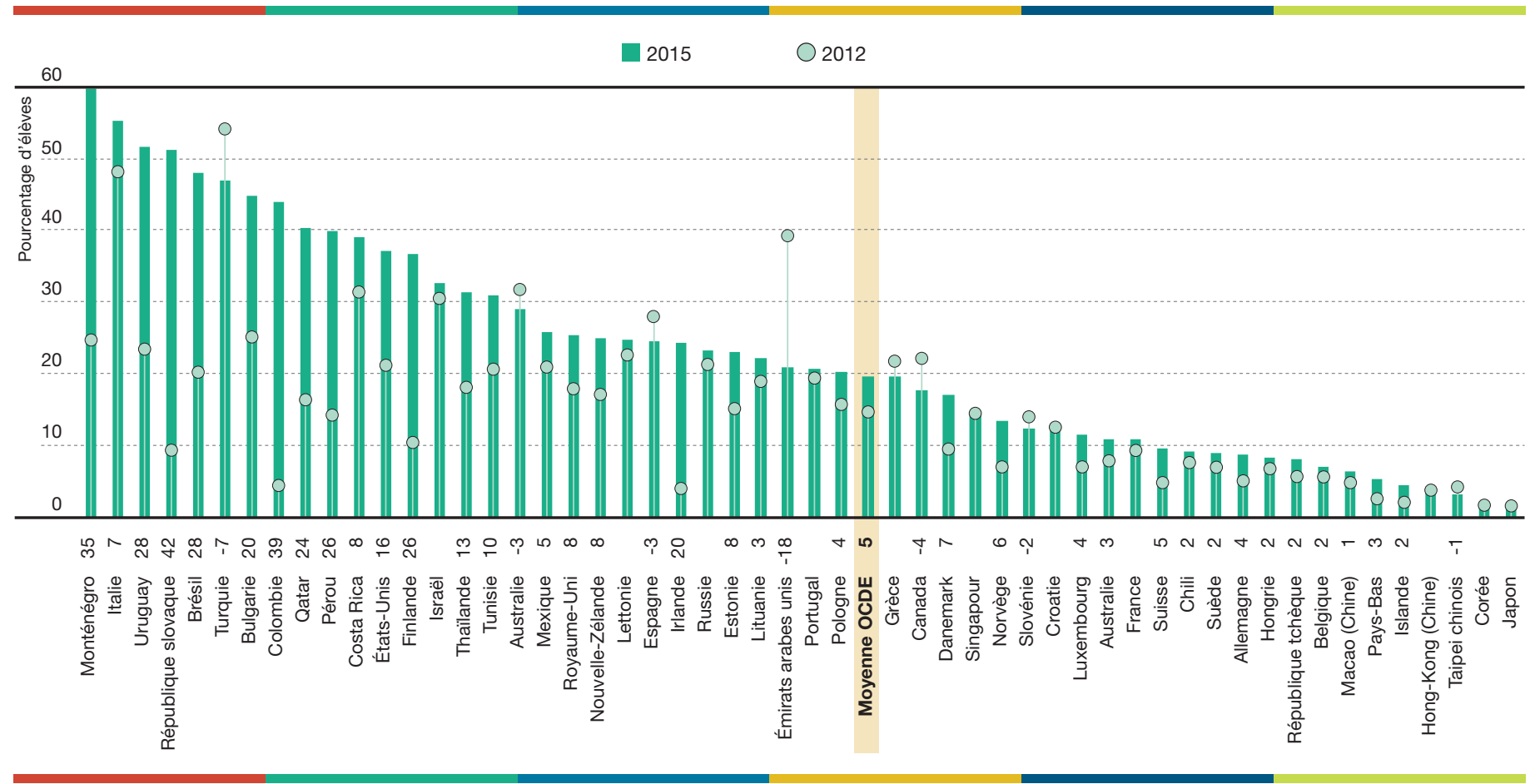

Remarques : Seuls sont inclus les pays et économies ayant participé à l'évaluation PISA en 2012 et en 2015.

Seules sont indiquées en regard du nom du pays/de l'économie les différences (exprimées en points de pourcentage) statistiquement significatives entre PISA 2012 et PISA 2015.

Les pays et économies sont classés par ordre décroissant du pourcentage d'élèves ayant séché une journée de cours au moins une fois durant les deux semaines précédant l'évaluation PISA, en 2015.

Source : OCDE, Base de données PISA 2015, tableaux II.3.1, II.3.2 et II.3.3. 


\section{Résultats fondés sur des analyses de niveau Système}

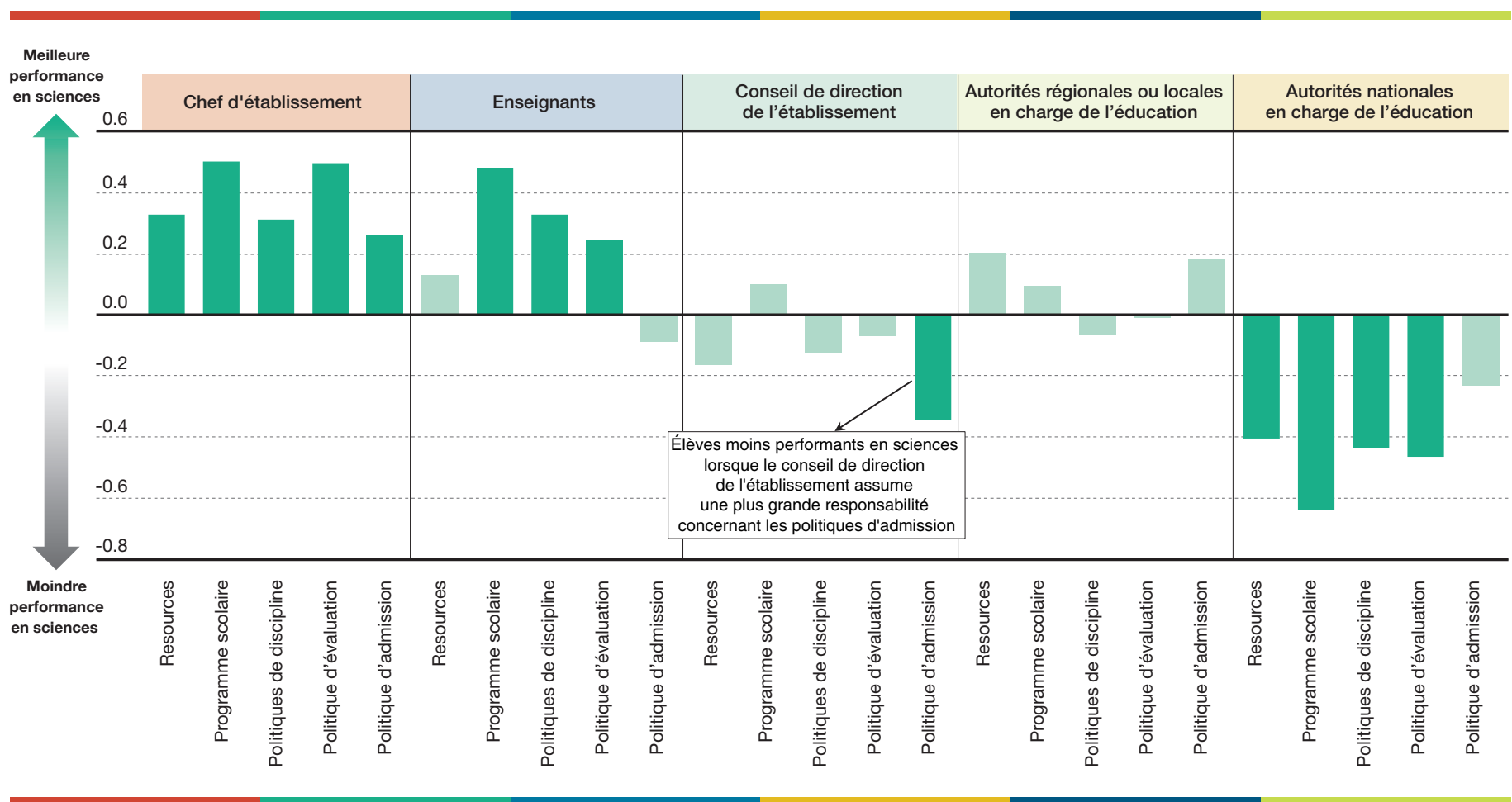

1. Les responsabilités concernant la gouvernance des établissements sont mesurées par la répartition (en pourcentage) des responsabilités en la matière, présentée dans le tableau ll.4.2 de la publication Résultats du PISA 2015 (Volume II) : Politiques et pratiques pour des établissements performants.

Remarques : Résultats fondés sur 70 systèmes d'éducation.

Les coefficients de corrélation statistiquement significatifs sont indiqués dans une couleur plus foncée.

Source : OCDE, Base de données PISA 2015

l'autonomie et la responsabilisation des établissements, une réalité déjà identifiée dans les précédentes évaluations PISA.

Les expériences et les travaux pratiques peuvent motiver les élèves et les aider à mieux comprendre les concepts scientifiques et à développer leurs compétences polyvalentes, telles que le raisonnement critique. Toutefois, pour que ces expériences et travaux pratiques soient réellement efficaces, les chefs d'établissement et les enseignants doivent y être correctement préparés. Les chefs d'établissement doivent s'assurer que le matériel de laboratoire se trouve en bon état et que les enseignants sont formés et encadrés en conséquence. Les enseignants doivent de leur côté concevoir des activités de laboratoire bien structurées qui rendent concrets les principaux concepts et grandes idées scientifiques, et permettent aux élèves d'établir des liens entre les travaux pratiques, les concepts scientifiques et les problèmes du monde réel. Les élèves doivent également prendre conscience qu'en prenant part à ces activités, ils manient aussi bien des objets que des concepts.

\section{Fournir un appui supplémentaire} aux établissements défavorisés.

L'apprentissage d'un élève ne devrait pas être affecté par le fait d'être issu d'une famille pauvre ou de l'immigration, d'être élevé dans une famille monoparentale ou de disposer de ressources limitées dans le foyer, comme ne pas avoir d'ordinateur ou une pièce au calme pour étudier. Les systèmes d'éducation performants l'ont bien compris et ont trouvé différentes façons d'affecter les ressources de manière à placer sur un pied d'égalité les élèves qui ne disposent pas des ressources humaines et matérielles dont bénéficient ceux des familles favorisées. Lorsque davantage d'élèves sont en situation d'apprentissage, c'est l'ensemble du système qui en bénéficie. Les résultats de l'enquête PISA ont mis en lumière un constat important : dans les pays et économies où davantage de ressources sont affectées aux établissements défavorisés, la performance globale des élèves en sciences est quelque peu supérieure, en particulier dans les pays de l'OCDE.

Les données PISA révèlent un certain nombre de différences entre les établissements favorisés et défavorisés, tant sur le plan qualitatif que quantitatif, qui dépeignent conjointement les environnements d'apprentissage radicalement différents de ces deux types d'établissements. Les établissements défavorisés comptent moins de professeurs de sciences qualifiés et sont moins susceptibles d'obliger les élèves à suivre des cours de sciences. Les élèves qui fréquentent ces établissements passent non seulement moins de temps en cours que les élèves des établissements favorisés, mais sont également moins exposés à un enseignement de qualité. Par exemple, les enseignants des établissements défavorisés sont moins susceptibles d'appliquer des stratégies pédagogiques efficaces, telles que l'explication ou la démonstration d'un concept scientifique. 
L'éventail de possibilités d'apprentissage en dehors des cours normaux est également bien plus limité dans les établissements défavorisés, qui tendent à proposer moins d'activités extrascolaires, comme les compétitions et les clubs de sciences, le sport, la musique et les activités artistiques. Ces établissements ont également tendance à être confrontés à davantage de problèmes de discipline et à un manque d'engagement de la part des élèves, qui se manifeste par le fait d'arriver en retard à l'école ou de sécher des journées de cours ; une réalité qui nuit aux opportunités d'apprentissage des élèves et à leur capacité à réussir à l'école. Certaines de ces différences entre les établissements favorisés et défavorisés sont amplifiées dans les pays qui pratiquent l'orientation précoce.

Les mesures compensatoires sont capitales et, de bien des façons, sont déjà en vigueur dans de nombreux pays. Des actions supplémentaires doivent cependant être menées. Par exemple, dans les établissements défavorisés, il ne suffit pas d'avoir à disposition davantage d'ordinateurs par élève ; encore faut-il que ces ordinateurs soient connectés à Internet et, surtout, qu'ils soient utilisés de façon à améliorer l'apprentissage, et non pas à le compromettre. Le fait que les élèves de ces établissements consacrent davantage de temps à étudier après leur journée de classe n'est pas suffisant ; ils doivent également passer plus de temps en cours et bénéficier d'un meilleur enseignement, à l'instar de leurs pairs des établissements favorisés. Ils ont également besoin de plus de soutien une fois la journée de classe terminée, sous la forme de cours particuliers ou d'activités extrascolaires enrichissantes, en particulier dans les pays et économies où les élèves des établissements favorisés consacrent plus de temps à étudier en dehors de leurs journées de classe, comme en Corée, en Croatie, en Italie, au Japon, à Macao (Chine) et au Taipei chinois. Les gouvernements peuvent avoir à mettre à disposition des ressources supplémentaires pour des cours particuliers gratuits dans les établissements défavorisés, afin d'empêcher le développement d'un système d'éducation parallèle et de garantir l'égalité des chances dans l'éducation.

À chaque problème sa solution, mais même lorsque différents établissements sont confrontés aux mêmes défis, il peut s'avérer nécessaire de mettre en œuvre des solutions adaptées en tirant parti des atouts déjà disponibles ; enfin, il convient d'assurer le suivi continu des progrès sur la voie de la réalisation des objectifs d'apprentissage.

\section{Évolution entre 2009 et 2015 des taux de redoublement}

Pourcentage d'élèves ayant déjà redoublé dans l'enseignement primaire, le premier cycle du secondaire ou le deuxième cycle du secondaire

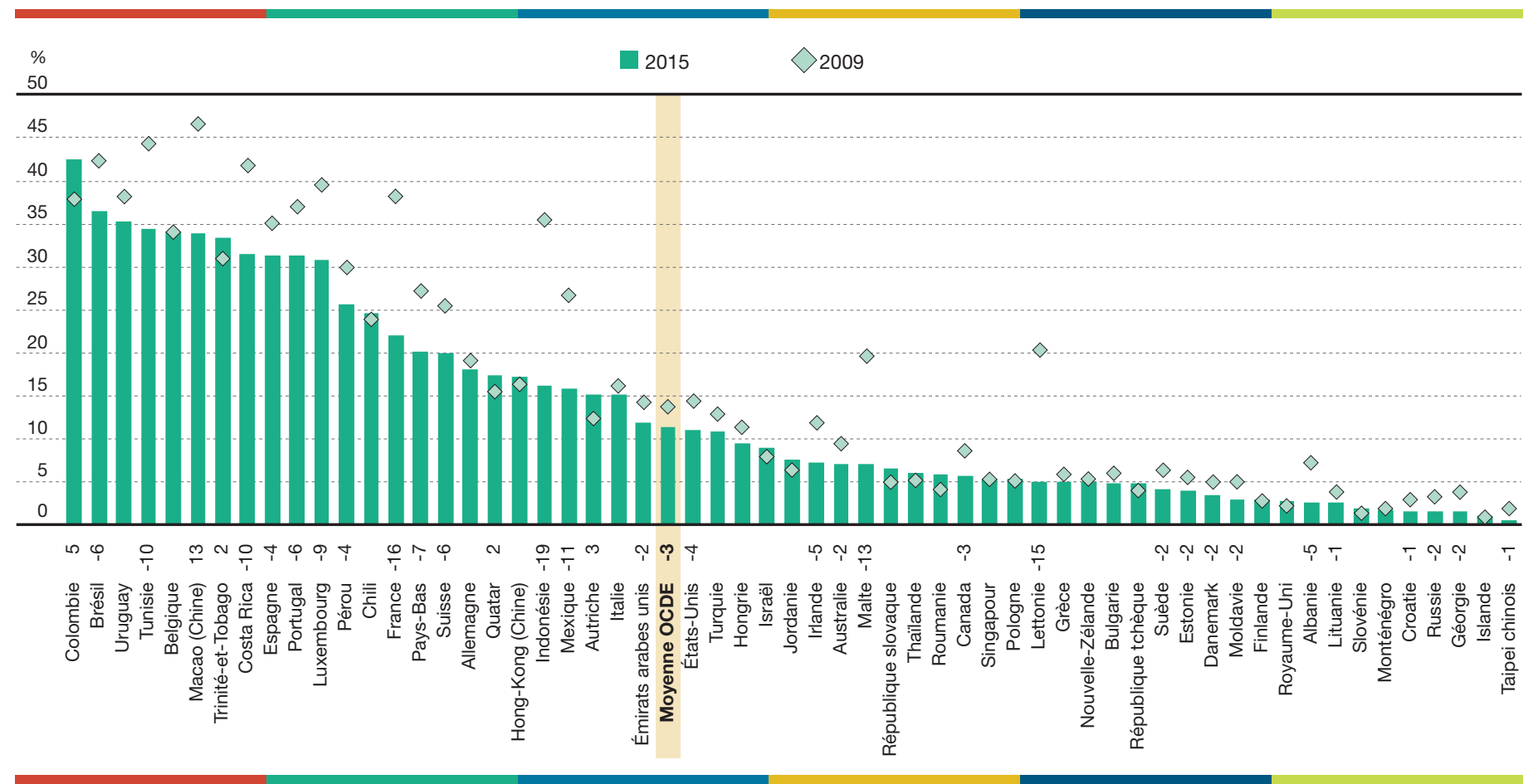

Remarques : Les différences statistiquement significatives sont indiquées en regard du nom du pays/de l'économie.

Seuls sont inclus les pays et économies disposant de données comparables pour PISA 2009 et PISA 2015.

Pour le Costa Rica, la Géorgie, Malte et la Moldavie, l'évolution entre PISA 2009 et PISA 2015 correspond à l'évolution entre 2010 et 2015 , car ces pays ont administré l'évaluation PISA 2009 en 2010 dans le cadre de PISA 2009+.

Les pays et économies sont classés par ordre décroissant du pourcentage d'élèves ayant déjà redoublé, en 2015.

Source : OCDE, Base de données PISA 2015, tableaux II.5.9, II.5.10 et II.5.11. 


\section{Résultats du PISA 2015}

Le Volume I, L'excellence et l'équité dans l'éducation, synthétise la performance des élèves dans l'enquête PISA 2015, et examine les indicateurs d'inclusion et d'égalité dans les systèmes d'éducation participants.

Le Volume II (à paraître), Politiques et pratiques pour des établissements performants, examine la relation entre la performance des élèves et diverses caractéristiques des établissements d'enseignement et des systèmes d'éducation.

Le Volume III (à paraître), Le bien-être des élèves, décrit les modes de vie et d'apprentissage des élèves.

Le Volume IV (à paraître), Les compétences des élèves en culture financière, analyse la façon dont les élèves de 15 ans comprennent les questions financières dans les 15 pays et économies qui ont administré ces épreuves facultatives.

Le Volume $\mathbf{V}$ (à paraître), La résolution collaborative de problèmes, analyse la capacité des élèves à travailler en binôme ou en groupe pour tenter de résoudre un problème.

Cet ouvrage est publié sous la responsabilité du Secrétaire général de l'OCDE. Les opinions et les interprétations exprimées ne reflètent pas nécessairement les vues des pays membres de l'OCDE.

Ce document et toute carte qu'il peut comprendre sont sans préjudice du statut de tout territoire, de la souveraineté s'exerçant sur ce dernier, du tracé des frontières et limites internationales, et du nom de tout territoire, ville ou région.

Les données statistiques concernant Israël sont fournies par et sous la responsabilité des autorités israéliennes compétentes. L'utilisation de ces données par l'OCDE est sans préjudice du statut des hauteurs du Golan, de Jérusalem-Est et des colonies de peuplement israéliennes en Cisjordanie aux termes du droit international.

P-S-J-G (Chine) fait référence aux quatre provinces chinoises participant à l'enquête PISA : Pékin, Shanghai, Jiangsu et Guangdong. CABA (Argentine) fait référence à la région Ciudad Autónoma de Buenos Aires, dont les données ont été adjugées.

ERYM fait référence à l'Ex-République yougoslave de Macédoine.

Russie fait référence à la Fédération de Russie.

Ce texte est disponible sous licence Attribution - Pas d'Utilisation Commerciale - Partage dans les Mêmes Conditions 3.0 Organisations Internationales (CC BY-NC-SA 3.0 IGO). Pour toute information spécifique quant à l'étendue et aux termes de la licence ainsi que d'une possible utilisation commercial de ce texte or pour toute usage de données PISA, prière de consulter les Conditions d'utilisation à http://www.oecd.org/fr/conditionsdutilisation. 
Pour tout complément d'information, contacter : Andreas Schleicher

Andreas.Schleicher@OECD.org

Consulter :

www.OECD.org/pisa 